\title{
Optimal proportional reinsurance with common shock dependence
}

\author{
Kam Chuen Yuen ${ }^{1}, \quad$ Zhibin Liang ${ }^{2 *}$ Ming Zhou ${ }^{3}$ \\ ${ }^{1}$ Department of Statistics and Actuarial Science, \\ The University of Hong Kong, Pokfulam Road, Hong Kong \\ ${ }^{2}$ School of Mathematical Sciences and Institute of Finance and Statistics, \\ Nanjing Normal University, Jiangsu 210023, China \\ ${ }^{3}$ China Institute for Actuarial Science, \\ Central University of Finance and Economics, Beijing 100081, China
}

${ }^{*}$ Corresponding author: Zhibin Liang, Email: liangzhibin111@hotmail.com. 


\begin{abstract}
In this paper, we consider the optimal proportional reinsurance strategy in a risk model with multiple dependent classes of insurance business, which extends the work of Liang and Yuen (2014) to the case with the reinsurance premium calculated under the expected value principle and to the model with two or more classes of dependent risks. Under the criterion of maximizing the expected exponential utility, closed-form expressions for the optimal strategies and value function are derived not only for the compound Poisson risk model but also for the diffusion approximation risk model. In particular, we find that the optimal reinsurance strategies under the expected value premium principle are very different from those under the variance premium principle in the diffusion risk model. The former depends not only on the safety loading, time and interest rate, but also on the claim size distributions and the counting processes, while the latter depends only on the safety loading, time and interest rate. Finally, numerical examples are presented to show the impact of model parameters on the optimal strategies.
\end{abstract}

Keywords: Dependent risks; Hamilton-Jacobi-Bellman equation; Compound Poisson process; Brownian motion; Exponential utility; Proportional reinsurance 


\section{Introduction}

In the actuarial literature, the problem of optimal reinsurance has been studied by many authors in the past two decades. Among others, Browne (1995), Schmidli (2001, 2002), Promislow and Young (2005), and Bai et al. (2013) derive optimal strategies that minimize ruin probability; Kaluszka $(2001,2004)$ examine the optimal reinsurance problem under various mean-variance premium principles; Centeno (1986, 2002), Hald and Schmidli (2004), and Liang and Guo (2008) focus on constructing optimal contracts that maximize the adjustment coefficient by the martingale approach; and Cai and Tan (2007), Bernard and Tian (2009), Chi and Meng (2014), and Cheung et al. (2014) investigate the optimal problem by adopting the criteria of minimizing tail risk measures such as value at risk and conditional tail expectation. In this paper, we study the optimal reinsurance problem with the objective that maximizes the expected utility of terminal wealth, which is a popular criterion for various optimization problems in finance and modern risk theory. See, for example, Irgens and Paulsen (2004), Yang and Zhang (2005), Liang et al. (2011), Liang and Yuen (2014), and references therein.

Suppose that an insurance company that has a portfolio of $m \geq 2$ dependent classes of insurance business such as auto insurance, casualty insurance, health insurance, life insurance, and so on. For the $l$ th $(l=1,2, \ldots, m)$ class of business, let $X_{i}^{(l)}(i=1,2, \ldots)$ be the claim size random variables following a common distribution $F_{l}(x)$ with $F_{l}(x)=0$ for $x \leq 0$ and $0<F_{l}(x)<1$ for $x>0$. Their means are denoted by $\mu_{l}=E\left(X^{(l)}\right)$. For each $l$, it is assumed that the moment generating function $M_{l}(r)=E\left(e^{r X^{(l)}}\right)$ exists. Then, the aggregate claims process for the $l$ th class of risk is given by

$$
S_{l}(t)=\sum_{i=1}^{\tilde{N}_{l}(t)} X_{i}^{(l)}
$$

where $\tilde{N}_{l}(t)$ is the claim number process for class $l$ for $l=1,2, \ldots, m$. As usual, it is assumed that $\left\{X_{i}^{(l)} ; l=1,2, \ldots, m, i=1,2, \ldots\right\}$ are independent claim size random variables, and that they are independent of $\left\{\tilde{N}_{l}(t) ; l=1,2, \ldots, m\right\}$.

In practice, the $m$ classes of insurance business are usually dependent in some way. A typical example is that an earthquake, hurricane or tsunami, often leads to various kinds of insurance claims such as medical claims, death claims, household claims, etc. This example demonstrates a practical scenario that a single event generates claims from different lines of insurance. To depict such a dependence structure among several classes of business, the so-called common shock risk model may be of some practical relevance. In this paper, we consider the risk model with common 
shock dependence in which the $m$ claim number processes are correlated in the way that

$$
\tilde{N}_{l}(t)=N_{l}(t)+N(t), \quad l=1,2, \ldots, m,
$$

with $N_{l}(t)$ and $N(t)$ being $m+1$ independent Poisson processes with intensities $\lambda_{1}, \ldots, \lambda_{m}$ and $\lambda$, respectively. It is obvious that the dependence among the $m$ classes of business is due to a common shock governed by the counting process $N(t)$. Therefore, the aggregate claims process generated from $m$ classes of business is given by

$$
S_{t}=\sum_{l=1}^{m} S_{l}(t)=\sum_{l=1}^{m}\left(\sum_{i=1}^{N_{l}(t)+N(t)} X_{i}^{(l)}\right) .
$$

For each class, we further assume that $E\left(X^{(l)} e^{r X^{(l)}}\right)=M_{l}^{\prime}(r)$ exists for $0<r<\zeta^{(l)}$, and that $\lim _{r \rightarrow \zeta^{(l)}} E\left(X^{(l)} e^{r X^{(l)}}\right)=\infty$ for some $0<\zeta^{(l)} \leq+\infty$.

Although research on risk models with dependent risk is increasing rapidly in recent years (see, for example, Wang (1998), Yuen et al. (2002, 2006), and the references therein), very few of these contributions deal with the problem in relation to optimal reinsurance. In the single period model, under the criterion of maximizing the expected utility of terminal wealth and that of maximizing the adjustment coefficient, Centeno (2005) studies the optimal excess of loss retention limits for two dependent classes of insurance risks. In the dynamic setting, Bai, Cai and Zhou (2013) also seek the optimal excess of loss reinsurance to minimize the ruin probability for the diffusion approximation risk model. Based on the aggregate claims process (1.1) with $m=2$, Liang and Yuen (2014) adopt the variance premium principle to study the optimal proportional reinsurance problem for both the compound Poisson risk model and the diffusion approximation risk model. Interestingly, their optimal results for the diffusion risk model show that the optimal reinsurance strategies of the two classes are the same, and that the claim size distributions as well as the counting processes have no effect on the optimal reinsurance strategy. These results motivate us to investigate the same optimization problem for the risk model with two or more classes of dependent risks, and the reinsurance premium is calculated according to the expected value principle, which is another popular premium principle in practice.

Based on this model set-up, the main objective of this paper is to further study the optimal reinsurance problem of Liang and Yuen (2014) by means of the expected value premium principle instead of the variance premium principle. Both the model extension and the change in reinsurance premium principle certainly make the problem more meaningful but also make it more difficult to 
tackle. Using the techniques of stochastic control theory and Hamilton-Jacobi-Bellman equation, we are still able to obtain explicit expressions for the optimal reinsurance strategies and the value function for both the compound Poisson case and the diffusion approximation case. In particular, we find that the optimal reinsurance strategies under the expected value principle are very different from those under variance principle in the diffusion approximation risk model. The former depends not only on the safety loading, time and interest rate, but also on the claim sizes and the claim number intensities, while the latter depends only on the safety loading, time and interest rate. It should be pointed out that the optimal reinsurance strategies with $m>2$ in the latter case still have the same form (see Section 6 and Liang and Yuen (2014) for details). Furthermore, we show that, with normal constraints on the control variables, the value function is still a classical solution to the corresponding Hamilton-Jacobi-Bellman equation. From the numerical examples, we can see that the optimal reinsurance strategies are more sensitive to the claim size distribution than to the counting processes. Also, the numerical results show that the optimal reinsurance strategy of one class of insurance risk is independent of the claim sizes of the other class when the claim sizes are exponentially distributed.

The rest of the paper is organized as follows. In Section 2, we introduce the model of study and give a rigorous mathematical formulation of the problem. In Sections 3 and 4, under the expected value premium principle, we discuss the optimal reinsurance strategies for the compound Poisson case as well as the diffusion approximation case, respectively. In Section 5, we present some numerical examples which show the impact of some model parameters on the optimal strategies. In Section 6, we extend the work of Liang and Yuen (2014) for the diffusion risk model to the case with $m>2$. Finally, we conclude the paper in Section 7 .

\section{Model and problem formulation}

As usual, we define the surplus process

$$
R_{t}=u+c t-S_{t}
$$

where $u$ is the amount of initial surplus, $c$ is the rate of premium, and $S_{t}$ is the aggregate claims process given by (1.1).

It was shown in Yuen et al. (2002) or Wang and Yuen (2005) that $S_{t}$ follows a compound Poisson

process with parameter $\tilde{\lambda}=\sum_{l=1}^{m} \lambda_{l}+\lambda$, and that the common distribution of the transformed 
claim size random variable $X^{\prime}$ is given by

$$
F_{X^{\prime}}(x)=\sum_{l=1}^{m} \frac{\lambda_{l}}{\tilde{\lambda}} F_{X^{(l)}}(x)+\frac{\lambda}{\tilde{\lambda}} F_{\sum_{l=1}^{m} X^{(l)}}(x) .
$$

Note that the compound Poisson process is a process with stationary and independent increment. Let $B_{t}$ be a standard Brownian motion. Then, the risk process

$$
\hat{S}_{t}=\left(\sum_{l=1}^{m} a_{l}\right) t-\sqrt{\sum_{l=1}^{m} \sigma_{l}^{2}+\sum_{i \neq j} \lambda \mu_{i} \mu_{j}} B_{t}
$$

can be seen as a diffusion approximation to the compound Poisson process $S_{t}$ (see Grandell (1991) for details), where

$$
a_{l}=\left(\lambda_{l}+\lambda\right) E\left(X^{(l)}\right), \quad \sigma_{l}^{2}=\left(\lambda_{l}+\lambda\right) E\left(\left(X^{(l)}\right)^{2}\right) .
$$

On the other hand, the Brownian motion with drift

$$
\hat{S}_{l}(t)=a_{l} t-\sigma_{l} B_{l t}
$$

can be seen as the diffusion approximation to compound Poisson process $S_{l}(t)$, where $B_{l t}(l=$ $1,2, \ldots, m)$ are standard Brownian motions with correlation coefficient $\rho_{i j}(i \neq j, i, j=1,2, \ldots, m)$. Furthermore,

$$
\sum_{l=1}^{m} \hat{S}_{l}(t)=\left(\sum_{l=1}^{m} a_{l}\right) t-\sum_{l=1}^{m} \sigma_{l} B_{l t}
$$

can be replaced by

$$
\sum_{l=1}^{m} \hat{S}_{l}(t)=\left(\sum_{l=1}^{m} a_{l}\right) t-\sqrt{\sum_{l=1}^{m} \sigma_{l}^{2}+\sum_{i \neq j} \sigma_{i} \sigma_{j} \rho_{i j} B_{t}},
$$

as the two forms have the same distributional properties. Hence, the sum $\sum_{l=1}^{m} \hat{S}_{l}(t)$ can also be regarded as a diffusion approximation to the compound Poisson process $S_{t}$ when

$$
\rho_{i j}=\frac{\lambda E\left(X^{(i)}\right) E\left(X^{(j)}\right)}{\sqrt{\left(\lambda_{i}+\lambda\right) E\left(\left(X^{(i)}\right)^{2}\right)\left(\lambda_{j}+\lambda\right) E\left(\left(X^{(j)}\right)^{2}\right)}}=\frac{\lambda \mu_{i} \mu_{j}}{\sigma_{i} \sigma_{j}},
$$

i.e., $E\left[B_{i t} B_{j t}\right]=\rho_{i j} t(i \neq j, i, j=1,2, \ldots, m)$.

In this paper, we allow the insurance company to continuously cede a fraction of its claims with the retention levels $q_{l t} \in[0,1]$ for each risk $X_{i}^{(l)}(l=1,2, \ldots, m)$, and the reinsurance premium rate at time $t$ is $\delta\left(q_{t}\right)$ with $q_{t}=\left(q_{1 t}, q_{2 t}, \ldots, q_{m t}\right)$. The company is also allowed to invest all its surplus in a risk-free asset (bond or bank account) with interest rate $r \geq 0$. Let $\left\{R_{t}^{q}, t \geq 0\right\}$ denote the 
associated surplus process, i.e., $R_{t}^{q}$ is the surplus of the insurer at time $t$, if the reinsurance strategy $q_{t}$ is adopted. This process then evolves as

$$
d R_{t}^{q}=\left[r R_{t}^{q}+\left(c-\delta\left(q_{t}\right)\right)\right] d t-\sum_{l=1}^{m} q_{l t} d S_{l}(t) .
$$

Replacing $S_{l}(t)(l=1,2, \ldots, m)$ of $(2.1)$ respectively by $\hat{S}_{l}(t)(l=1,2, \ldots, m)$ yield a new surplus process

$$
d \hat{R}_{t}^{q}=\left[r \hat{R}_{t}^{q}+\left(c-\delta\left(q_{t}\right)\right)-\sum_{l=1}^{m} q_{l t} a_{l}\right] d t+\sum_{l=1}^{m} q_{l t} \sigma_{l} d B_{l t}
$$

or equivalently,

$$
\begin{aligned}
d \hat{R}_{t}^{q}= & {\left[r \hat{R}_{t}^{q}+\left(c-\delta\left(q_{t}\right)\right)-\sum_{l=1}^{m} q_{l t} a_{l}\right] d t } \\
& +\sqrt{\sum_{l=1}^{m} \sigma_{l}^{2} q_{l t}^{2}+\sum_{i \neq j} q_{i t} q_{j t} \lambda \mu_{i} \mu_{j}} d B_{t} .
\end{aligned}
$$

Assume now that the insurer is interested in maximizing the expected utility of terminal wealth, say at time $T$. The utility function is $u(x)$, which satisfies $u^{\prime}>0$ and $u^{\prime \prime}<0$. Then, the objective function is

$$
J^{q}(t, x)=E\left[u\left(R_{T}^{q}\right) \mid R_{t}^{q}=x\right]
$$

or

$$
J^{q}(t, x)=E\left[u\left(\hat{R}_{T}^{q}\right) \mid \hat{R}_{t}^{q}=x\right]
$$

We shall discuss (2.3) and (2.4) separately, and hence the use of the same notation $J^{q}(t, x)$ will not cause any confusion. The corresponding value function is then given by

$$
V(t, x)=\sup _{q} J^{q}(t, x)
$$

We further assume that the insurer has an exponential utility function

$$
u(x)=-\frac{\varrho}{\nu} e^{-\nu x}
$$

where $\varrho>0$ and $\nu>0$. This utility has constant absolute risk aversion (CARA) parameter $\nu$. Such utility function plays an important role in insurance mathematics and actuarial practice, since they are the only functions under which the principle of " zero utility" gives a fair premium that is independent of the level of reserves of an insurance company (see Gerber (1979)).

Let $C^{1,2}$ denote the space of $\phi(t, x)$ such that $\phi$ and its partial derivatives $\phi_{t}, \phi_{x}, \phi_{x x}$ are continuous on $[0, T] \times R$. To solve the above problem, we use the dynamic programming approach 
described in Fleming and Soner (2006). It follows from the standard arguments that if the value function $V \in C^{1,2}$, then $V$ satisfies the following Hamilton-Jacobi-Bellman (HJB) equation

$$
\sup _{q} \mathcal{A}^{q} V(t, x)=0
$$

for $t<T$, with the boundary condition

$$
V(T, x)=u(x)
$$

where

$$
\begin{aligned}
\mathcal{A}^{q} V(t, x)=V_{t} & +[r x+c-\delta(q)] V_{x} \\
& +\sum_{l=1}^{m} \lambda_{l} E\left[V\left(t, x-q_{l} X^{(l)}\right)-V(t, x)\right] \\
& +\lambda E\left[V\left(t, x-\sum_{l=1}^{m} q_{l} X^{(l)}\right)-V(t, x)\right],
\end{aligned}
$$

for the risk process $(2.1)$, and

$$
\begin{aligned}
\mathcal{A}^{q} V(t, x)=V_{t} & +\left[r x+c-\delta(q)-\sum_{l=1}^{m} q_{l t} a_{l}\right] V_{x} \\
& +\frac{1}{2}\left(\sum_{l=1}^{m} \sigma_{l}^{2} q_{l t}^{2}+\sum_{i \neq j} q_{i t} q_{j t} \lambda \mu_{i} \mu_{j}\right) V_{x x},
\end{aligned}
$$

for the risk process $(2.2)$.

Applying the methods of Fleming and Soner (2006) and Yang and Zhang (2005), we have the following verification theorem:

Theorem 2.1. Let $W \in C^{1,2}$ be a classical solution to (2.6) that satisfies (2.7). Then, the value function $V$ of (2.5) coincides with $W$. That is,

$$
W(t, x)=V(t, x)
$$

Furthermore, let $q^{*}$ be such that

$$
\mathcal{A}^{q^{*}} V(t, x)=0
$$

holds for all $(t, x) \in[0, T) \times R$, then

$$
q^{*}\left(t, R_{t}^{*}\right)=\left(q_{1}^{*}\left(t, R_{t}^{*}\right), q_{2}^{*}\left(t, R_{t}^{*}\right), \ldots, q_{m}^{*}\left(t, R_{t}^{*}\right)\right)
$$

is the optimal strategy, where $R_{t}^{*}$ is the surplus process under the optimal strategy.

Remark 2.1. In this paper, continuous trading is allowed; all assets are infinitely divisible; the process $R_{t}^{q}$ is well defined on a complete probability space $(\Omega, \mathcal{F}, P)$; the information at time $t$ is given by the complete filtration $\mathcal{F}_{t}$ generated by $R_{t}^{q}$; and the strategy $q_{t}$ is $\mathcal{F}_{t}$-predictable. 


\section{Optimal results for the compound Poisson risk model}

In this section, we consider the optimization problem for the risk model (2.1). Throughout Sections 3 and 4 , we assume that the reinsurance premium is calculated according to the expected value principle. That is,

$$
\delta(q)=\sum_{l=1}^{m}\left(1+\eta_{l}\right)\left(1-q_{l}\right) a_{l}
$$

where $\eta_{l}(l=1,2, \ldots, m)$ are the reinsurer's safety loading of the $m$ classes of insurance business.

To solve the equation

$$
\begin{aligned}
\sup _{q}\left\{V_{t}+\right. & {[r x+c-\delta(q)] V_{x}+\sum_{l=1}^{m} \lambda_{l} E\left[V\left(t, x-q_{l} X^{(l)}\right)-V(t, x)\right] } \\
+ & \left.\lambda E\left[V\left(t, x-\sum_{l=1}^{m} q_{l} X^{(l)}\right)-V(t, x)\right]\right\}=0
\end{aligned}
$$

with the boundary condition $V(T, x)=u(x)$, we apply the method of Browne (1995) to fit a solution of the form

$$
V(t, x)=-\frac{\varrho}{\nu} \exp \left[-\nu x e^{r(T-t)}+h(T-t)\right]
$$

where $h(\cdot)$ is a suitable function such that (3.2) is a solution to (2.6). The boundary condition $V(T, x)=u(x)$ implies $h(0)=0$.

From (3.2), we get

$$
\left\{\begin{array}{l}
V_{t}=V(t, x)\left[\nu x r e^{r(T-t)}-h^{\prime}(T-t)\right], \\
V_{x}=V(t, x)\left[-\nu e^{r(T-t)}\right], \\
V_{x x}=V(t, x)\left[\nu^{2} e^{2 r(T-t)}\right], \\
E\left[V\left(t, x-q_{l} X^{(l)}\right)-V(t, x)\right]=V(t, x)\left[M_{l}\left(\nu q_{l} e^{r(T-t)}\right)-1\right] \\
E\left[V\left(t, x-\sum_{l=1}^{m} q_{l} X^{(l)}\right)-V(t, x)\right] \\
=V(t, x)\left[\prod_{l=1}^{m} M_{l}\left(\nu q_{l} e^{r(T-t)}\right)-1\right] .
\end{array}\right.
$$

Then, using (2.6) and (3.3), we obtain

$$
\begin{aligned}
\inf _{q} \quad\{- & h^{\prime}(T-t)-c \nu e^{r(T-t)}-\sum_{l=1}^{m} \lambda_{l}-\lambda+\delta(q) \nu e^{r(T-t)} \\
& \left.+\sum_{l=1}^{m} \lambda_{l} M_{l}\left(\nu q_{l} e^{r(T-t)}\right)+\lambda \prod_{l=1}^{m} M_{l}\left(\nu q_{l} e^{r(T-t)}\right)\right\}=0,
\end{aligned}
$$

for $t<T$. Let

$$
\tilde{f}(q)=\delta(q) \nu e^{r(T-t)}+\sum_{l=1}^{m} \lambda_{l} M_{l}\left(\nu q_{l} e^{r(T-t)}\right)+\lambda \prod_{l=1}^{m} M_{l}\left(\nu q_{l} e^{r(T-t)}\right) .
$$


For any $t \in[0, T]$, we have

$$
\left\{\begin{aligned}
\frac{\partial \tilde{f}(q)}{\partial q_{l}} & =\left(-\left(1+\eta_{l}\right) a_{l}+M_{l}^{\prime}\left(\nu q_{l} e^{r(T-t)}\right)\left(\lambda_{l}+\lambda \prod_{j \neq l} M_{j}\left(\nu q_{j} e^{r(T-t)}\right)\right)\right) \cdot \nu e^{r(T-t)}, \\
\frac{\partial^{2} \tilde{f}(q)}{\partial q_{l}^{2}} & =M_{l}^{\prime \prime}\left(\nu q_{l} e^{r(T-t)}\right)\left(\lambda_{l}+\lambda \prod_{j \neq l} M_{j}\left(\nu q_{j} e^{r(T-t)}\right)\right) \cdot \nu^{2} e^{2 r(T-t)}>0, \\
\frac{\partial^{2} \tilde{f}(q)}{\partial q_{l} \partial q_{k}} & \left.=\lambda M_{l}^{\prime}\left(\nu q_{l} e^{r(T-t)}\right) M_{k}^{\prime}\left(\nu q_{k} e^{r(T-t)}\right) \prod_{j \neq l, k} M_{j}\left(\nu q_{j} e^{r(T-t)}\right)\right) \cdot \nu^{2} e^{2 r(T-t)},
\end{aligned}\right.
$$

for $l \neq k, l, k=1,2, \ldots, m$, where $M_{l}^{\prime \prime}(r)=E\left(\left(X^{(l)}\right)^{2} e^{r X^{(l)}}\right)$.

Lemma 3.1. For $l=1,2, \ldots, m, \tilde{f}(q)$ of (3.5) is a convex function with respect to $q_{l}$.

Proof: To prove that $\tilde{f}(q)$ is a convex function with respect to $q_{l}$ for each $l$, it is sufficient to prove that the Hessian matrix of $\tilde{f}(q)$ is positive definite.

Let

$$
\overline{\mathbf{A}}=\left(\begin{array}{cccc}
\lambda_{1} M_{1}^{\prime \prime}(\cdot) & 0 & \ldots & 0 \\
0 & \lambda_{2} M_{2}^{\prime \prime}(\cdot) & \ldots & 0 \\
\vdots & \vdots & \ddots & \vdots \\
0 & 0 & \ldots & \lambda_{m} M_{m}^{\prime \prime}(\cdot)
\end{array}\right)
$$

and

$$
\overline{\mathbf{B}}=\left(\begin{array}{cccc}
\frac{M_{1}^{\prime \prime}(\cdot)}{M_{1}(\cdot)} & \frac{M_{1}^{\prime}(\cdot) M_{2}^{\prime}(\cdot)}{M_{1}(\cdot) M_{2}(\cdot)} & \ldots & \frac{M_{1}^{\prime}(\cdot) M_{m}^{\prime}(\cdot)}{M_{1}(\cdot) M_{m}(\cdot)} \\
\frac{M_{2}^{\prime}(\cdot) M_{1}^{\prime}(\cdot)}{M_{2}(\cdot) M_{1}(\cdot)} & \frac{M_{2}^{\prime \prime}(\cdot)}{M_{2}(\cdot)} & \ldots & \frac{M_{2}^{\prime}(\cdot) M_{m}^{\prime}(\cdot)}{M_{2}(\cdot) M_{m}(\cdot)} \\
\vdots & \vdots & \ddots & \vdots \\
\frac{M_{m}^{\prime}(\cdot) M_{1}^{\prime}(\cdot)}{M_{m}(\cdot) M_{1}(\cdot)} & \frac{M_{m}^{\prime}(\cdot) M_{2}^{\prime}(\cdot)}{M_{m}(\cdot) M_{2}(\cdot)} & \ldots & \frac{M_{m}^{\prime \prime}(\cdot)}{M_{m}(\cdot)}
\end{array}\right) .
$$

Then, the Hessian matrix of $\tilde{f}(q)$ can be written as

$$
\left(\begin{array}{cccc}
\frac{\partial^{2} \tilde{f}(q)}{\partial q_{1}^{2}} & \frac{\partial^{2} \tilde{f}(q)}{\partial q_{1} \partial q_{2}} & \cdots & \frac{\partial^{2} \tilde{f}(q)}{\partial q_{1} \partial q_{m}} \\
\frac{\partial^{2} \tilde{f}(q)}{\partial q_{2} \partial q_{1}} & \frac{\partial^{2} \tilde{f}(q)}{\partial q_{2}^{2}} & \ldots & \frac{\partial^{2} \tilde{f}(q)}{\partial q_{2} \partial q_{m}} \\
\vdots & \vdots & \ddots & \vdots \\
\frac{\partial^{2} \tilde{f}(q)}{\partial q_{m} \partial q_{1}} & \frac{\partial^{2} \tilde{f}(q)}{\partial q_{m} \partial q_{2}} & \ldots & \frac{\partial^{2} \tilde{f}(q)}{\partial q_{m}^{2}}
\end{array}\right)=\left(\overline{\mathbf{A}}+\lambda \prod_{j=1}^{m} M_{j}(\cdot) \cdot \overline{\mathbf{B}}\right) \cdot \nu^{2} e^{2 r(T-t)}
$$

It is clear that $\overline{\mathbf{A}}$ is a positive definite matrix. Moreover, for any $C=\left(c_{1}, c_{2}, \ldots, c_{m}\right) \in R^{n}$ and 
$C \neq 0$, we have

$$
\begin{aligned}
C \cdot \overline{\mathbf{B}} \cdot C^{\prime} & =\sum_{i=1}^{m} c_{i}^{2} \frac{M_{i}^{\prime \prime}(\cdot)}{M_{i}(\cdot)}+\sum_{i \neq k} 2 c_{i} c_{k} \frac{M_{i}^{\prime}(\cdot) M_{k}^{\prime}(\cdot)}{M_{i}(\cdot) M_{k}(\cdot)} \\
& =\sum_{i=1}^{m} c_{i}^{2} \frac{M_{i}^{\prime \prime}(\cdot) M_{i}(\cdot)}{\left(M_{i}(\cdot)\right)^{2}}+\sum_{i \neq k} 2 c_{i} c_{k} \frac{M_{i}^{\prime}(\cdot)}{M_{i}(\cdot)} \cdot \frac{M_{k}^{\prime}(\cdot)}{M_{k}(\cdot)} \\
& \geq \sum_{i=1}^{m} c_{i}^{2}\left(\frac{M_{i}^{\prime}(\cdot)}{M_{i}(\cdot)}\right)^{2}+\sum_{i \neq k} 2 c_{i} c_{k} \frac{M_{i}^{\prime}(\cdot)}{M_{i}(\cdot)} \cdot \frac{M_{k}^{\prime}(\cdot)}{M_{k}(\cdot)} \\
& =\left(\sum_{i=1}^{m} c_{i} \frac{M_{i}^{\prime}(\cdot)}{M_{i}(\cdot)}\right)^{2}>0,
\end{aligned}
$$

where $C^{\prime}$ is the transpose of $C$, and the first inequality follows from the Cauchy-Schwarz inequality. Hence, $\overline{\mathbf{B}}$ is also a positive definite matrix. As a result, the Hessian matrix is positive definite.

It is easy to see that the minimizer $\left(q_{1}(T-t), q_{2}(T-t), \ldots, q_{m}(T-t)\right)$ of $\tilde{f}(q)$ satisfies the following equations

$$
-\left(1+\eta_{l}\right) a_{l}+M_{l}^{\prime}\left(\nu q_{l} e^{r(T-t)}\right)\left(\lambda_{l}+\lambda \prod_{j \neq l} M_{j}\left(\nu q_{j} e^{r(T-t)}\right)\right)=0, \quad l=1,2, \ldots, m .
$$

Moreover, we have

Lemma 3.2. For any $t \in[0, T]$, if both $\hat{q}=\left(\hat{q}_{1}, \hat{q}_{2}, \ldots, \hat{q}_{m}\right)$ and $\tilde{q}=\left(\tilde{q}_{1}, \tilde{q}_{2}, \ldots, \tilde{q}_{m}\right)$ are the solutions to the equation (3.6), then $\hat{q}=\tilde{q}$.

Proof: $\quad$ Assume that $\hat{q} \neq \tilde{q}$. Let

$$
\tilde{f}^{\prime}(q)=\left(\frac{\partial \tilde{f}(q)}{\partial q_{1}}, \frac{\partial \tilde{f}(q)}{\partial q_{2}}, \ldots, \frac{\partial \tilde{f}(q)}{\partial q_{m}}\right),
$$

and

$$
\tilde{f}^{\prime \prime}(q)=\left(\begin{array}{cccc}
\frac{\partial^{2} \tilde{f}(q)}{\partial q_{1}^{2}} & \frac{\partial^{2} \tilde{f}(q)}{\partial q_{1} \partial q_{2}} & \ldots & \frac{\partial^{2} \tilde{f}(q)}{\partial q_{1} \partial q_{m}} \\
\frac{\partial^{2} \tilde{f}(q)}{\partial q_{2} \partial q_{1}} & \frac{\partial^{2} \tilde{f}(q)}{\partial q_{2}^{2}} & \cdots & \frac{\partial^{2} \tilde{f}(q)}{\partial q_{2} \partial q_{m}} \\
\vdots & \vdots & \ddots & \vdots \\
\frac{\partial^{2} \tilde{f}(q)}{\partial q_{m} \partial q_{1}} & \frac{\partial^{2} \tilde{f}(q)}{\partial q_{m} \partial q_{2}} & \ldots & \frac{\partial^{2} \tilde{f}(q)}{\partial q_{m}^{2}}
\end{array}\right) .
$$

By Taylor's Theorem in higher dimensions, we have

$$
\begin{aligned}
\tilde{f}(\hat{q}) & =\tilde{f}(\tilde{q})+\tilde{f}^{\prime}(\tilde{q}) \cdot(\hat{q}-\tilde{q})^{\prime}+\frac{1}{2}(\hat{q}-\tilde{q}) \cdot \tilde{f}^{\prime \prime}(\tilde{q}+\theta(\hat{q}-\tilde{q})) \cdot(\hat{q}-\tilde{q})^{\prime} \\
& =\tilde{f}(\tilde{q})+\frac{1}{2} \Delta_{1},
\end{aligned}
$$

where $\theta \in[0,1]$, and

$$
\Delta_{1}=(\hat{q}-\tilde{q}) \cdot \tilde{f}^{\prime \prime}(\tilde{q}+\theta(\hat{q}-\tilde{q})) \cdot(\hat{q}-\tilde{q})^{\prime}
$$


From Lemma 3.1, we know that the Hessian matrix $f^{\prime \prime}(q)$ is a positive definite matrix. Thus, $\Delta_{1}>0$ for any $\hat{q}-\tilde{q} \neq 0$. This implies that

$$
\tilde{f}(\hat{q})=\tilde{f}(\tilde{q})+\frac{1}{2} \Delta_{1}>\tilde{f}(\tilde{q})
$$

Along the same lines, one can show that $\tilde{f}(\tilde{q})>\tilde{f}(\hat{q})$, which is contrary to (3.7).

With the help of Lemma 3.2, and using the elementary fixed-point theorem (see for example, Ortega and Rheinboldt (1970)), one can show the uniqueness and the existence of the solution to the equation (3.6).

For $m=2$, the equation (3.6) becomes

$$
\left\{\begin{array}{l}
-\left(1+\eta_{1}\right) a_{1}+M_{1}^{\prime}\left(n_{1}\right)\left(\lambda_{1}+\lambda M_{2}\left(n_{2}\right)\right)=0 \\
-\left(1+\eta_{2}\right) a_{2}+M_{2}^{\prime}\left(n_{2}\right)\left(\lambda_{2}+\lambda M_{1}\left(n_{1}\right)\right)=0
\end{array}\right.
$$

where $n_{1}=\nu q_{1} e^{r(T-t)}$ and $n_{2}=\nu q_{2} e^{r(T-t)}$. In addition, we have

Lemma 3.3. Let $M_{i}^{-1}\left(M_{i}^{\prime-1}\right)$ be the inverse function of $M_{i}\left(M_{i}^{\prime}\right)$ for $i=1,2$. If the following inequalities hold:

$$
\begin{cases}M_{2}^{-1}\left(1+\eta_{1}+\eta_{1} \cdot \frac{\lambda_{1}}{\lambda}\right) & >M_{2}^{\prime-1}\left(\left(1+\eta_{2}\right) \mu_{2}\right), \\ M_{1}^{-1}\left(1+\eta_{2}+\eta_{2} \cdot \frac{\lambda_{2}}{\lambda}\right) & >M_{1}^{\prime-1}\left(\left(1+\eta_{1}\right) \mu_{1}\right),\end{cases}
$$

or

$$
\left\{\begin{array}{cc}
M_{2}^{-1}\left(1+\eta_{1}+\eta_{1} \cdot \frac{\lambda_{1}}{\lambda}\right) & <M_{2}^{\prime-1}\left(\left(1+\eta_{2}\right) \mu_{2}\right) \\
M_{1}^{-1}\left(1+\eta_{2}+\eta_{2} \cdot \frac{\lambda_{2}}{\lambda}\right) & <M_{1}^{\prime-1}\left(\left(1+\eta_{1}\right) \mu_{1}\right)
\end{array}\right.
$$

then the equation (3.8) has a unique positive root $\left(\bar{n}_{1}, \bar{n}_{2}\right)$.

Proof: Let

$$
H_{1}\left(n_{1}, n_{2}\right)=-\left(1+\eta_{1}\right) a_{1}+M_{1}^{\prime}\left(n_{1}\right)\left(\lambda_{1}+\lambda M_{2}\left(n_{2}\right)\right)
$$

and

$$
H_{2}\left(n_{1}, n_{2}\right)=-\left(1+\eta_{2}\right) a_{2}+M_{2}^{\prime}\left(n_{2}\right)\left(\lambda_{2}+\lambda M_{1}\left(n_{1}\right)\right)
$$

Assume that the equation $H_{1}\left(n_{1}, n_{2}\right)=0$ implies $n_{2}=f_{1}\left(n_{1}\right)$, and that $H_{2}\left(n_{1}, n_{2}\right)=0$ implies $n_{2}=f_{2}\left(n_{1}\right)$. Denote by $f_{i}^{-1}$ the inverse function of $f_{i}$ for $i=1,2$. Differentiating both sides of the equation $H_{1}\left(n_{1}, n_{2}\right)=0$ with respect to $n_{1}$ yields

$$
\lambda f_{1}^{\prime}\left(n_{1}\right) M_{2}^{\prime}\left(n_{2}\right) M_{1}^{\prime}\left(n_{1}\right)+\left(\lambda M_{2}\left(n_{2}\right)+\lambda_{1}\right) M_{1}^{\prime \prime}\left(n_{1}\right)=0
$$


and thus

$$
f_{1}^{\prime}\left(n_{1}\right)=-\frac{\left(\lambda M_{2}\left(n_{2}\right)+\lambda_{1}\right) M_{1}^{\prime \prime}\left(n_{1}\right)}{\lambda M_{2}^{\prime}\left(n_{2}\right) M_{1}^{\prime}\left(n_{1}\right)}<0 .
$$

Therefore, the function $f_{1}\left(n_{1}\right)$ is a decreasing function with

$$
\left\{\begin{array}{l}
f_{1}(0)=M_{2}^{-1}\left(1+\eta_{1}+\eta_{1} \cdot \frac{\lambda_{1}}{\lambda}\right)>M_{2}^{-1}(1)=0 \\
f_{1}^{-1}(0)=M_{1}^{\prime-1}\left(\left(1+\eta_{1}\right) \mu_{1}\right)>M_{1}^{\prime-1}\left(\mu_{1}\right)=0
\end{array}\right.
$$

By the same token, one can use $H_{2}\left(n_{1}, n_{2}\right)=0$ to obtain

$$
f_{2}^{\prime}\left(n_{1}\right)=-\frac{\lambda M_{2}^{\prime}\left(n_{2}\right) M_{1}^{\prime}\left(n_{1}\right)}{\left(\lambda M_{1}\left(n_{1}\right)+\lambda_{2}\right) M_{2}^{\prime \prime}\left(n_{2}\right)}<0
$$

and thus the function $f_{2}\left(n_{1}\right)$ is also a decreasing function with

$$
\left\{\begin{array}{l}
f_{2}(0)=M_{2}^{\prime-1}\left(\left(1+\eta_{2}\right) \mu_{2}\right)>M_{2}^{\prime-1}\left(\mu_{2}\right)=0 \\
f_{2}^{-1}(0)=M_{1}^{-1}\left(1+\eta_{2}+\eta_{2} \cdot \frac{\lambda_{2}}{\lambda}\right)>M_{1}^{-1}(1)=0 .
\end{array}\right.
$$

Therefore, if the following inequalities hold

$$
\begin{cases}f_{1}(0) & >f_{2}(0) \\ f_{1}^{-1}(0) & <f_{2}^{-1}(0)\end{cases}
$$

or

$$
\left\{\begin{array}{l}
f_{1}(0) \quad<f_{2}(0) \\
f_{1}^{-1}(0)<f_{2}^{-1}(0)
\end{array}\right.
$$

the functions $f_{1}\left(n_{1}\right)$ and $f_{2}\left(n_{1}\right)$ have at least one point of intersection at some $\bar{n}_{1}>0$. Then, it follows from Lemma 3.2 that the equation (3.8) has a unique positive root. That is, the equation (3.8) has a unique positive root $\left(\bar{n}_{1}, \bar{n}_{2}\right)$ with $\bar{n}_{2}=f_{1}\left(\bar{n}_{1}\right)=f_{2}\left(\bar{n}_{1}\right)$.

From Lemma 3.3, we obtain

$$
\bar{n}_{1}=\nu q_{1}(T-t) e^{r(T-t)}, \quad \text { and } \quad \bar{n}_{2}=\nu q_{2}(T-t) e^{r(T-t)},
$$

which in turn give

$$
\left\{\begin{array}{l}
q_{1}(T-t)=\frac{\bar{n}_{1}}{\nu} e^{-r(T-t)}, \\
q_{2}(T-t)=\frac{\bar{n}_{2}}{\nu} e^{-r(T-t)}
\end{array}\right.
$$


It is not difficult to see from (3.8) that $\bar{n}_{1}$ and $\bar{n}_{2}$ are constants depending on the safety loadings $\eta_{1}$ and $\eta_{2}$, the claim size distributions as well as the counting processes.

Let

$$
t_{1}=T-\frac{1}{r} \ln \frac{\bar{n}_{1}}{\nu} \quad \text { for } \quad \nu<\bar{n}_{1}<\nu e^{r T} ; \quad t_{2}=T-\frac{1}{r} \ln \frac{\bar{n}_{2}}{\nu} \quad \text { for } \quad \nu<\bar{n}_{2}<\nu e^{r T}
$$

For $\bar{n}_{1} \leq \nu\left(\bar{n}_{2} \leq \nu\right)$, we set $t_{1}=T\left(t_{2}=T\right)$; and for $\bar{n}_{1} \geq \nu e^{r T}\left(\bar{n}_{2} \geq \nu e^{r T}\right)$, we set $t_{1}=0\left(t_{2}=0\right)$. To make sure that the retention level $q_{1 t}, q_{2 t} \in[0,1]$, we need to discuss the optimal values in the following two cases: (1) $\bar{n}_{1} \leq \bar{n}_{2}$; and (2) $\bar{n}_{1} \geq \bar{n}_{2}$.

Case 1: In this case, we have $t_{1} \geq t_{2}$. When $0 \leq t \leq t_{2}$, we have $\left(q_{1 t}^{*}, q_{1 t}^{*}\right)=\left(q_{1}(T-t), q_{2}(T-t)\right)$. Denote the $h$ function in (3.4) by $h_{1}$. Substituting $\left(q_{1}(T-t), q_{2}(T-t)\right)$ into (3.4), we get

$$
h_{1}(T-t)=\tilde{h}_{1}(T-t)+C_{1},
$$

where

$$
\begin{aligned}
\tilde{h}_{1}(T-t)= & \frac{1}{r}\left[\left(1+\eta_{1}\right) a_{1}+\left(1+\eta_{2}\right) a_{2}-c\right] \nu e^{r(T-t)} \\
& -\left(\lambda_{1}+\lambda_{2}+\lambda+\left(1+\eta_{1}\right) a_{1} \bar{n}_{1}+\left(1+\eta_{2}\right) a_{2} \bar{n}_{2}\right)(T-t) \\
& +\left(\lambda_{1} M_{1}\left(\bar{n}_{1}\right)+\lambda_{2} M_{2}\left(\bar{n}_{2}\right)+\lambda M_{1}\left(\bar{n}_{1}\right) M_{2}\left(\bar{n}_{2}\right)\right)(T-t),
\end{aligned}
$$

and $C_{1}$ is a constant that will be determined later.

For $t \geq t_{2}, q_{2}(T-t) \geq 1$, and thus $q_{2 t}^{*}=1$. Substituting $q_{2 t}^{*}=1$ into (3.4), we get

$$
\begin{aligned}
\inf _{q_{1}} \quad\{ & -h^{\prime}(T-t)-c \nu e^{r(T-t)}-\lambda_{l}-\lambda_{2}-\lambda+\delta\left(q_{1}\right) \nu e^{r(T-t)} \\
& +\lambda_{2} M_{2}\left(\nu e^{r(T-t)}\right)+M_{1}\left(\nu q_{1} e^{r(T-t)}\right)\left(\lambda_{1}+\lambda M_{2}\left(\nu e^{r(T-t)}\right)\right\}=0,
\end{aligned}
$$

for $t<T$, where $\delta\left(q_{1}\right)=\left(1+\eta_{1}\right)\left(1-q_{1}\right) a_{1}$. Therefore, the minimizer of the equation (3.11) is

$$
\hat{q}_{1}(T-t)=M_{1}^{\prime-1}\left[\frac{\left(1+\eta_{1}\right) a_{1}}{\lambda_{1}+\lambda M_{2}\left(\nu e^{r(T-t)}\right)}\right] \cdot \frac{1}{\nu} e^{-r(T-t)} .
$$

As $M_{1}^{\prime}(x)$ is an increasing function of $x$, it is not difficult to see that $\hat{q}_{1}(T-t)$ is an increasing function of $t$.

Denote by $t_{01}$ the solution to the equation $\hat{q}_{1}(T-t)=1$, and by $h_{2}$ the $h$ function in (3.4). For $t_{2} \leq t \leq t_{01},\left(q_{1 t}^{*}, q_{2 t}^{*}\right)=\left(\hat{q}_{1}(T-t), 1\right)$. Substituting $\left(\hat{q}_{1}(T-t), 1\right)$ into $(3.4)$, we get

$$
h_{2}(T-t)=\tilde{h}_{2}(T-t)+C_{2},
$$


where

$$
\begin{aligned}
\tilde{h}_{2}(T-t)= & \frac{1}{r}\left[\left(1+\eta_{1}\right) a_{1}-c\right] \nu e^{r(T-t)}-\left(\lambda_{1}+\lambda_{2}+\lambda\right)(T-t)-\int_{0}^{T-t}\left(1+\eta_{1}\right) a_{1} \hat{q}_{1}(s) \nu e^{r s} d s \\
& +\int_{0}^{T-t} \lambda_{2} M_{2}\left(\nu e^{r s}\right)+M_{1}\left(\hat{q}_{1}(s) \nu e^{r s}\right)\left(\lambda_{1}+\lambda M_{2}\left(\nu e^{r s}\right)\right) d s
\end{aligned}
$$

and $C_{2}$ is also a constant that will be determined later.

For $t_{01} \leq t \leq T,\left(q_{1 t}^{*}, q_{2 t}^{*}\right)=(1,1)$. Denote the $h$ function in $(3.4)$ by $h_{3}$. Then, putting $(1,1)$ into (3.4), we get

$$
\begin{gathered}
h_{3}(T-t)=-\frac{1}{r} c \nu\left(e^{r(T-t)}-1\right)-\left(\lambda_{1}+\lambda_{2}+\lambda\right)(T-t) \\
+\int_{0}^{T-t}\left(\lambda_{1} M_{1}\left(\nu e^{r s}\right)+\lambda_{2} M_{2}\left(\nu e^{r s}\right)\right. \\
\left.+\lambda M_{1}\left(\nu e^{r s}\right) M_{2}\left(\nu e^{r s}\right)\right) d s .
\end{gathered}
$$

Let

$$
C_{1}=h_{2}\left(T-t_{2}\right)-\tilde{h}_{1}\left(T-t_{2}\right), \quad \text { and } \quad C_{2}=h_{3}\left(T-t_{01}\right)-\tilde{h}_{2}\left(T-t_{01}\right) .
$$

Then, we have

$$
\left\{\begin{array}{l}
h_{2}\left(T-t_{2}\right)=\tilde{h}_{1}\left(T-t_{2}\right)+C_{1}=h_{1}\left(T-t_{2}\right), \\
h_{3}\left(T-t_{01}\right)=\tilde{h}_{2}\left(T-t_{01}\right)+C_{2}=h_{2}\left(T-t_{01}\right) .
\end{array}\right.
$$

Case 2: In this case, we have $t_{1} \leq t_{2}$. For $0 \leq t \leq t_{1},\left(q_{1 t}^{*}, q_{12}^{*}\right)=\left(q_{1}(T-t), q_{2}(T-t)\right)$ from which the $h$ function in $(3.4)$ can be written as

$$
h_{4}(T-t)=\tilde{h}_{1}(T-t)+C_{4} .
$$

For $t \geq t_{1}$, we have $q_{1}(T-t) \geq 1$, and thus $q_{1 t}^{*}=1$. Then, similar to the derivation of (3.12), we get the minimizer

$$
\hat{q}_{2}(T-t)=M_{2}^{\prime-1}\left[\frac{\left(1+\eta_{2}\right) a_{2}}{\lambda_{2}+\lambda M_{1}\left(\nu e^{r(T-t)}\right.}\right] \cdot \frac{1}{\nu} e^{-r(T-t)}
$$

which is increasing function of $t$.

Denote by $t_{02}$ the solution to the equation $\hat{q}_{2}(T-t)=1$, and by $h_{5}$ the $h$ function in (3.4). For $t_{1} \leq t \leq t_{02},\left(q_{1 t}^{*}, q_{1 t}^{*}\right)=\left(1, \hat{q}_{1}(T-t)\right)$. It follows from $(3.4)$ and $\left(q_{1 t}^{*}, q_{1 t}^{*}\right)=\left(1, \hat{q}_{1}(T-t)\right)$ that

$$
h_{5}(T-t)=\tilde{h}_{5}(T-t)+C_{5},
$$


where

$$
\begin{aligned}
\tilde{h}_{5}(T-t)= & \frac{1}{r}\left[\left(1+\eta_{2}\right) a_{2}-c\right] \nu e^{r(T-t)}-\left(\lambda_{1}+\lambda_{2}+\lambda\right)(T-t)-\int_{0}^{T-t}\left(1+\eta_{2}\right) a_{2} \hat{q}_{2}(s) \nu e^{r s} d s \\
& +\int_{0}^{T-t} \lambda_{1} M_{1}\left(\nu e^{r s}\right)+M_{2}\left(\hat{q}_{2}(s) \nu e^{r s}\right)\left(\lambda_{2}+\lambda M_{1}\left(\nu e^{r s}\right)\right) d s
\end{aligned}
$$

For $t_{02} \leq t \leq T,\left(q_{1 t}^{*}, q_{1 t}^{*}\right)=(1,1)$, and the corresponding $h$ function in (3.4) is $h_{3}$ of (3.14). Letting

$$
C_{4}=h_{5}\left(T-t_{1}\right)-\tilde{h}_{1}\left(T-t_{1}\right), \quad \text { and } \quad C_{5}=h_{3}\left(T-t_{02}\right)-\tilde{h}_{5}\left(T-t_{02}\right),
$$

yields

$$
\left\{\begin{array}{l}
h_{5}\left(T-t_{1}\right)=\tilde{h}_{1}\left(T-t_{1}\right)+C_{4}=h_{4}\left(T-t_{1}\right), \\
h_{3}\left(T-t_{02}\right)=\tilde{h}_{5}\left(T-t_{02}\right)+C_{5}=h_{5}\left(T-t_{02}\right) .
\end{array}\right.
$$

The following theorem summarizes the above analysis.

Theorem 3.1. Let $\left(\bar{n}_{1}, \bar{n}_{2}\right)$ be the unique positive root of the equation (3.6), and $\hat{q}_{1}(T-t), \hat{q}_{2}(T-t)$ and $\left(q_{1}(T-t), q_{2}(T-t)\right)$ be given in (3.12), (3.16) and (3.9), respectively. Recall the functions $h_{1}(T-t), h_{2}(T-t), h_{3}(T-t), h_{4}(T-t)$ and $h_{5}(T-t)$ defined in (3.10), (3.13), (3.14), (3.15) and (3.17), respectively. Then, we have

(i) If Case (1) holds, i.e., $\bar{n}_{1} \leq \bar{n}_{2}$, for any $t \in[0, T]$, the optimal reinsurance strategies $q_{1 t}^{*}$ and $q_{2 t}^{*}$ under the model (2.1) are

$$
\left(q_{1 t}^{*}, q_{2 t}^{*}\right)= \begin{cases}\left(q_{1}(T-t), q_{2}(T-t)\right), & 0 \leq t \leq t_{2}, \\ \left(\hat{q}_{1}(T-t), 1\right), & t_{2} \leq t \leq t_{01}, \\ (1,1), & t_{01} \leq t \leq T,\end{cases}
$$

and the value function $V(t, x)$ is given by

$$
V(t, x)= \begin{cases}-\frac{\varrho}{\nu} \exp \left\{-\nu x e^{r(T-t)}+h_{1}(T-t)\right\}, & 0 \leq t \leq t_{2}, \\ -\frac{\varrho}{\nu} \exp \left\{-\nu x e^{r(T-t)}+h_{2}(T-t)\right\}, & t_{2} \leq t \leq t_{01}, \\ -\frac{\varrho}{\nu} \exp \left\{-\nu x e^{r(T-t)}+h_{3}(T-t)\right\}, & t_{01} \leq t \leq T ;\end{cases}
$$


(ii) If Case (2) holds, i.e., $\bar{n}_{1} \geq \bar{n}_{2}$, for any $t \in[0, T]$, the optimal reinsurance strategies under the model (2.1) are

$$
\left(q_{1 t}^{*}, q_{2 t}^{*}\right)= \begin{cases}\left(q_{1}(T-t), q_{2}(T-t)\right), & 0 \leq t \leq t_{1}, \\ \left(1, \hat{q}_{2}(T-t)\right), & t_{1} \leq t \leq t_{02} \\ (1,1), & t_{02} \leq t \leq T\end{cases}
$$

and the value function is given by

$$
V(t, x)= \begin{cases}-\frac{\varrho}{\nu} \exp \left\{-\nu x e^{r(T-t)}+h_{4}(T-t)\right\}, & 0 \leq t \leq t_{1}, \\ -\frac{\varrho}{\nu} \exp \left\{-\nu x e^{r(T-t)}+h_{5}(T-t)\right\}, & t_{1} \leq t \leq t_{02}, \\ -\frac{\varrho}{\nu} \exp \left\{-\nu x e^{r(T-t)}+h_{3}(T-t)\right\}, & t_{02} \leq t \leq T .\end{cases}
$$

Remark 3.1. Since

$$
\left\{\begin{array}{l}
h_{1}\left(T-t_{2}\right)=h_{2}\left(T-t_{2}\right), \\
h_{2}\left(T-t_{1}\right)=h_{3}\left(T-t_{1}\right), \\
h_{4}\left(T-t_{1}\right)=h_{5}\left(T-t_{1}\right), \\
h_{5}\left(T-t_{2}\right)=h_{3}\left(T-t_{2}\right),
\end{array}\right.
$$


$V(t, x)$ is a continuous function on $[0, T] \times R$. Furthermore,

$$
\left\{\begin{aligned}
h_{1}^{\prime}\left(T-t_{2}\right)= & -c \bar{n}_{2}-\left(\lambda_{1}+\lambda_{2}+\lambda+\left(1+\eta_{1}\right) a_{1}\left(\bar{n}_{1}-\bar{n}_{2}\right)\right. \\
& +\lambda_{1} M_{1}\left(\bar{n}_{1}\right)+M_{2}\left(\bar{n}_{2}\right)\left(\lambda_{2}+\lambda M_{1}\left(\bar{n}_{1}\right)\right) \\
= & h_{2}^{\prime}\left(T-t_{2}\right), \\
h_{2}^{\prime}\left(T-t_{01}\right)= & -c \nu e^{r\left(T-t_{01}\right)}-\left(\lambda_{1}+\lambda_{2}+\lambda\right)+\lambda_{1} M_{1}\left(\nu e^{r\left(T-t_{01}\right)}\right) \\
& +M_{2}\left(\nu e^{r\left(T-t_{01}\right)}\right)\left(\lambda_{2}+\lambda M_{1}\left(\nu e^{r\left(T-t_{01}\right)}\right)\right) \\
= & h_{3}^{\prime}\left(T-t_{01}\right), \\
h_{4}^{\prime}\left(T-t_{1}\right)= & -c \bar{n}_{1}-\left(\lambda_{1}+\lambda_{2}+\lambda+\left(1+\eta_{2}\right) a_{2}\left(\bar{n}_{2}-\bar{n}_{1}\right)\right. \\
& +\lambda_{1} M_{1}\left(\bar{n}_{1}\right)+M_{2}\left(\bar{n}_{2}\right)\left(\lambda_{2}+\lambda M_{1}\left(\bar{n}_{1}\right)\right) \\
= & h_{5}^{\prime}\left(T-t_{1}\right), \\
= & -c \nu e^{r\left(T-t_{02}\right)}-\left(\lambda_{1}+\lambda_{2}+\lambda\right)+\lambda_{2} M_{2}\left(\nu e^{r\left(T-t_{02}\right)}\right) \\
& +M_{1}\left(\nu e^{r\left(T-t_{02}\right)}\right)\left(\lambda_{1}+\lambda M_{2}\left(\nu e^{r\left(T-t_{02}\right)}\right)\right) \\
= & h_{3}^{\prime}\left(T-t_{02}\right) .
\end{aligned}\right.
$$

Therefore, we have $V(t, x) \in C^{1,2}$. That is, $V(t, x)$ is a classical solution to the HJB equation (2.6).

Remark 3.2. From (3.9) or the numerical examples in Section 5, we can see that the optimal reinsurance strategies in the compound Poisson risk model depend not only on the safety loading, time and interest rate, but also on the claim size distributions and the counting processes. Such a result is consistent with the one obtained under the variance principle (see Section 5 of Liang and Yuen (2014)). However, for the diffusion risk model, the result obtained in the following section is different from that obtained under the variance principle.

For $m>2$, a note is given at the end of Section 4, i.e., Remark 4.3. 


\section{Optimal results for the diffusion model}

In this section, we discuss the optimization problem for the diffusion approximation risk model. Under the expected value principle, the surplus process of the model (2.2) evolves as

$$
\begin{aligned}
d \hat{R}_{t}^{q}= & {\left[r \hat{R}_{t}^{q}+\left(c-\delta\left(q_{t}\right)\right)-\sum_{l=1}^{m} q_{l t} a_{l}\right] d t } \\
& +\sqrt{\sum_{l=1}^{m} \sigma_{l}^{2} q_{l t}^{2}+\sum_{i \neq j} q_{i t} q_{j t} \lambda \mu_{i} \mu_{j}} d B_{t},
\end{aligned}
$$

and the HJB equation of this problem is

$$
\begin{aligned}
\sup _{q}\{ & V_{t}+\left[r x+c-\delta(q)-\sum_{l=1}^{m} q_{l} a_{l}\right] V_{x} \\
& \left.+\frac{1}{2}\left(\sum_{l=1}^{m} \sigma_{l}^{2} q_{l}^{2}+\sum_{i \neq j} q_{i} q_{j} \lambda \mu_{i} \mu_{j}\right) V_{x x}\right\}=0,
\end{aligned}
$$

for $t<T$, with the boundary condition $V(T, x)=u(x)$. Again, we consider a solution with the form of (3.2). After substituting (3.3) into (4.1) and some algebraic manipulation, the equation (4.1) becomes

$$
\begin{aligned}
\inf _{q}\{ & -h^{\prime}(T-t)-\left[c-\delta(q)-\sum_{l=1}^{m} q_{l} a_{l}\right] \nu e^{r(T-t)} \\
& \left.+\frac{1}{2}\left(\sum_{l=1}^{m} \sigma_{l}^{2} q_{l}^{2}+\sum_{i \neq j} q_{i} q_{j} \lambda \mu_{i} \mu_{j}\right) \nu^{2} e^{2 r(T-t)}\right\}=0 .
\end{aligned}
$$

Let

$$
\begin{aligned}
\tilde{g}(q)= & \left(\delta(q)+\sum_{l=1}^{m} q_{l} a_{l}\right) \nu e^{r(T-t)} \\
& +\frac{1}{2}\left(\sum_{l=1}^{m} \sigma_{l}^{2} q_{l}^{2}+\sum_{i \neq j} q_{i} q_{j} \lambda \mu_{i} \mu_{j}\right) \nu^{2} e^{2 r(T-t)} .
\end{aligned}
$$

Then, for any $t \in[0, T]$, we have

$$
\left\{\begin{aligned}
\frac{\partial \tilde{g}(q)}{\partial q_{l}} & =-\eta_{l} a_{l} \nu e^{r(T-t)}+\left(q_{l} \sigma_{l}^{2}+\lambda \sum_{i \neq l} q_{i} \mu_{l} \mu_{i}\right) \nu^{2} e^{2 r(T-t)} \\
\frac{\partial^{2} \tilde{g}(q)}{\partial q_{l}^{2}} & =\sigma_{l}^{2} \nu^{2} e^{2 r(T-t)}>0 \\
\frac{\partial^{2} \tilde{g}(q)}{\partial q_{l} \partial q_{k}} & =\lambda \mu_{l} \mu_{k} \nu^{2} e^{2 r(T-t)}>0
\end{aligned}\right.
$$

for $l \neq k, l, k=1,2, \ldots, m$.

Lemma 4.1. For $l=1,2, \ldots, m, \tilde{g}(q)$ of (4.3) is a convex function with respect to $q_{l}$.

Proof: To prove the lemma, it is sufficient to prove the Hessian matrix of $\tilde{g}(q)$ is positive definite. 
Define

$$
\mathbf{A}=\left(\begin{array}{ccccc}
\sigma_{1}^{2} & \lambda \mu_{1} \mu_{2} & \lambda \mu_{1} \mu_{3} & \ldots & \lambda \mu_{1} \mu_{m} \\
\lambda \mu_{2} \mu_{1} & \sigma_{2}^{2} & \lambda \mu_{2} \mu_{3} & \ldots & \lambda \mu_{2} \mu_{m} \\
\lambda \mu_{3} \mu_{1} & \lambda \mu_{3} \mu_{2} & \sigma_{3}^{2} & \ldots & \lambda \mu_{3} \mu_{m} \\
\vdots & \vdots & \vdots & \ddots & \vdots \\
\lambda \mu_{m} \mu_{1} & \lambda \mu_{m} \mu_{2} & \lambda \mu_{m} \mu_{3} & \ldots & \sigma_{m}^{2}
\end{array}\right) .
$$

For any $C=\left(c_{1}, c_{2}, \ldots, c_{m}\right) \in R^{n}$ and $C \neq 0$, we have

$$
\begin{aligned}
C \cdot \mathbf{A} \cdot C^{\prime} & =\sum_{i=1}^{m} c_{i}^{2} \sigma_{i}^{2}+\sum_{i \neq k} 2 c_{i} c_{k} \lambda \mu_{i} \mu_{k} \\
& =\sum_{i=1}^{m} c_{i}^{2}\left(\lambda_{i}+\lambda\right) E\left(X^{(i)}\right)^{2}+\sum_{i \neq k} 2 c_{i} c_{k} \lambda E\left(X^{(i)}\right) E\left(X^{(k)}\right) \\
& =\sum_{i=1}^{m} c_{i}^{2} \lambda_{i} E\left(X^{(i)}\right)^{2}+\lambda\left\{\sum_{i=1}^{m} c_{i}^{2} E\left(X^{(i)}\right)^{2}+\sum_{i \neq k} 2 c_{i} c_{k} E\left(X^{(i)}\right) E\left(X^{(k)}\right)\right\} \\
& \geq \sum_{i=1}^{m} c_{i}^{2} \lambda_{i} E\left(X^{(i)}\right)^{2}+\lambda\left\{\sum_{i=1}^{m} c_{i}^{2}\left(E\left(X^{(i)}\right)\right)^{2}+\sum_{i \neq k} 2 c_{i} c_{k} E\left(X^{(i)}\right) E\left(X^{(k)}\right)\right\} \\
& =\sum_{i=1}^{m} c_{i}^{2} \lambda_{i} E\left(X^{(i)}\right)^{2}+\lambda\left(\sum_{i=1}^{m} c_{i} E\left(X^{(i)}\right)\right)^{2}>0,
\end{aligned}
$$

which implies that $\mathbf{A}$ is a positive definite matrix. Since the Hessian matrix in this case is given by

$$
\left(\begin{array}{cccc}
\frac{\partial^{2} \tilde{g}(q)}{\partial q_{1}^{2}} & \frac{\partial^{2} \tilde{g}(q)}{\partial q_{1} \partial q_{2}} & \cdots & \frac{\partial^{2} \tilde{g}(q)}{\partial q_{1} \partial q_{m}} \\
\frac{\partial^{2} \tilde{g}(q)}{\partial q_{2} \partial q_{1}} & \frac{\partial^{2} \tilde{g}(q)}{\partial q_{2}^{2}} & \cdots & \frac{\partial^{2} \tilde{g}(q)}{\partial q_{2} \partial q_{m}} \\
\vdots & \vdots & \ddots & \vdots \\
\frac{\partial^{2} \tilde{g}(q)}{\partial q_{m} \partial q_{1}} & \frac{\partial^{2} \tilde{g}(q)}{\partial q_{m} \partial q_{2}} & \cdots & \frac{\partial^{2} \tilde{g}(q)}{\partial q_{m}^{2}}
\end{array}\right)=\mathbf{A} \cdot \nu^{2} e^{2 r(T-t)}
$$

the Hessian matrix is also a positive definite matrix.

It is clear that the minimizer $\bar{q}(T-t)=\left(\bar{q}_{1}(T-t), \bar{q}_{2}(T-t), \ldots, \bar{q}_{m}(T-t)\right)$ of $(4.2)$ satisfies the following equations

$$
-\eta_{l} a_{l} \nu e^{r(T-t)}+\left(q_{l} \sigma_{l}^{2}+\lambda \sum_{i \neq l} q_{i} \mu_{l} \mu_{i}\right) \nu^{2} e^{2 r(T-t)}=0, \quad l=1,2, \ldots, m
$$

Or equivalently,

$$
\mathbf{A} q^{\prime}=\mathbf{B}
$$


where

$$
\mathbf{B}=\left(\begin{array}{l}
\eta_{1} a_{1} \\
\eta_{2} a_{2} \\
\eta_{3} a_{3} \\
\vdots \\
\eta_{m} a_{m}
\end{array}\right) \frac{1}{\nu} e^{-r(T-t)}
$$

Since the matrix $\mathbf{A}$ is a positive definite matrix which is invertible, the equation (4.4) has a unique positive solution

$$
\bar{q}(T-t)=\left(\mathbf{A}^{-1} \mathbf{B}\right)^{\prime}
$$

For $m=2$, the equation (4.4) becomes

$$
\left\{\begin{array}{l}
q_{1} \sigma_{1}^{2}+\lambda q_{2} \mu_{1} \mu_{2}=\frac{\eta_{1} a_{1}}{\nu} e^{-r(T-t)}, \\
q_{2} \sigma_{2}^{2}+\lambda q_{1} \mu_{1} \mu_{2}=\frac{\eta_{2} a_{2}}{\nu} e^{-r(T-t)},
\end{array}\right.
$$

it is easy to show that the solution to this equation is

$$
\left\{\begin{array}{l}
\bar{q}_{1}(T-t)=\frac{a_{1} \eta_{1} \sigma_{2}^{2}-\lambda \mu_{1} \mu_{2} a_{2} \eta_{2}}{\sigma_{1}^{2} \sigma_{2}^{2}-\lambda^{2} \mu_{1}^{2} \mu_{2}^{2}} \cdot \frac{1}{\nu} e^{-r(T-t)} \\
\bar{q}_{2}(T-t)=\frac{a_{2} \eta_{2} \sigma_{1}^{2}-\lambda \mu_{1} \mu_{2} a_{1} \eta_{1}}{\sigma_{1}^{2} \sigma_{2}^{2}-\lambda^{2} \mu_{1}^{2} \mu_{2}^{2}} \cdot \frac{1}{\nu} e^{-r(T-t)}
\end{array}\right.
$$

Let

$$
\left\{\begin{array}{l}
A_{1}=\frac{a_{1} \eta_{1} \sigma_{2}^{2}-\lambda \mu_{1} \mu_{2} a_{2} \eta_{2}}{\sigma_{1}^{2} \sigma_{2}^{2}-\lambda^{2} \mu_{1}^{2} \mu_{2}^{2}} \\
A_{2}=\frac{a_{2} \eta_{2} \sigma_{1}^{2}-\lambda \mu_{1} \mu_{2} a_{1} \eta_{1}}{\sigma_{1}^{2} \sigma_{2}^{2}-\lambda^{2} \mu_{1}^{2} \mu_{2}^{2}}
\end{array}\right.
$$

and

$$
t_{3}=T-\frac{1}{r} \ln \frac{A_{1}}{\nu} \quad \text { for } \quad \nu<A_{1}<\nu e^{r T} ; \quad t_{4}=T-\frac{1}{r} \ln \frac{A_{2}}{\nu} \quad \text { for } \quad \nu<A_{2}<\nu e^{r T} .
$$

For $A_{1} \leq \nu\left(A_{2} \leq \nu\right)$, we set $t_{3}=T\left(t_{4}=T\right) ;$ and for $A_{1} \geq \nu e^{r T}\left(A_{2} \geq \nu e^{r T}\right)$, we set $t_{3}=0$ $\left(t_{4}=0\right)$. To discuss the optimal values, we first prove the following lemma which plays a key role in this section.

Lemma 4.1. Given $\lambda$ and $\mu_{i}, a_{i}, \sigma_{i}$ for $i=1,2$, we have

$$
\frac{\lambda \mu_{1} \mu_{2} a_{2}}{\sigma_{2}^{2} a_{1}} \leq \frac{\lambda \mu_{1} \mu_{2} a_{2}+\sigma_{1}^{2} a_{2}}{\sigma_{2}^{2} a_{1}+\lambda \mu_{1} \mu_{2} a_{1}} \leq \frac{\sigma_{1}^{2} a_{2}}{\lambda \mu_{1} \mu_{2} a_{1}} .
$$


Proof: Using Cauchy-Schwarz inequality, one can show that $\sigma_{i}^{2}>\lambda \mu_{1} \mu_{2}$ for $i=1$, 2 , and thus

$$
\frac{\lambda \mu_{1} \mu_{2} a_{2}}{\sigma_{2}^{2} a_{1}} \leq \frac{\sigma_{1}^{2} a_{2}}{\lambda \mu_{1} \mu_{2} a_{1}} .
$$

In addition, for any positive parameters $a, b, c$ and $d$, if

$$
\frac{a}{b} \leq \frac{c}{d}
$$

then we have

$$
\frac{a}{b} \leq \frac{a+c}{b+d} \leq \frac{c}{d}
$$

which completes the proof.

To make sure that the optimal reinsurance strategies belong to the interval $[0,1]$, we need to investigate the optimal results in the following four cases:

(I)

$$
\frac{\lambda \mu_{1} \mu_{2} a_{2}}{\sigma_{2}^{2} a_{1}} \eta_{2} \leq \eta_{1} \leq \frac{\lambda \mu_{1} \mu_{2} a_{2}+\sigma_{1}^{2} a_{2}}{\sigma_{2}^{2} a_{1}+\lambda \mu_{1} \mu_{2} a_{1}} \eta_{2}
$$

$$
\frac{\lambda \mu_{1} \mu_{2} a_{2}+\sigma_{1}^{2} a_{2}}{\sigma_{2}^{2} a_{1}+\lambda \mu_{1} \mu_{2} a_{1}} \eta_{2} \leq \eta_{1} \leq \frac{\sigma_{1}^{2} a_{2}}{\lambda \mu_{1} \mu_{2} a_{1}} \eta_{2}
$$

$$
\eta_{1} \leq \frac{\lambda \mu_{1} \mu_{2} a_{2}}{\sigma_{2}^{2} a_{1}} \eta_{2}
$$

$$
\eta_{1} \geq \frac{\sigma_{1}^{2} a_{2}}{\lambda \mu_{1} \mu_{2} a_{1}} \eta_{2}
$$

Case I: In this case, we have $\bar{q}_{1}(T-t)>0, \bar{q}_{2}(T-t)>0$, and $A_{1} \leq A_{2}$. So, we have $t_{3} \geq t_{4}$. Also, for $0 \leq t \leq t_{4}$, we have $\left(q_{1 t}^{*}, q_{2 t}^{*}\right)=\left(\bar{q}_{1}(T-t), \bar{q}_{2}(T-t)\right)$. Then, substituting $\left(\bar{q}_{1}(T-t), \bar{q}_{2}(T-t)\right)$ into (4.2), we get

$$
h_{6}(T-t)=\tilde{h}_{6}(T-t)+C_{6},
$$

where

$$
\begin{aligned}
\tilde{h}_{6}(T-t)= & \frac{1}{r}\left(\left(1+\eta_{1}\right) a_{1}+\left(1+\eta_{2}\right) a_{2}-c\right) \nu e^{r(T-t)} \\
& +\left(\frac{1}{2} \sigma_{1}^{2} A_{1}^{2}+\frac{1}{2} \sigma_{2}^{2} A_{2}^{2}+A_{1} A_{2} \lambda \mu_{1} \mu_{2}-a_{1} \eta_{1} A_{1}-a_{2} \eta_{2} A_{2}\right)(T-t),
\end{aligned}
$$

and $C_{6}$ is a constant that will be determined later. 
For $t \geq t_{4}, \bar{q}_{2}(T-t) \geq 1$, and thus $q_{2 t}^{*}=1$. Substituting $q_{2 t}^{*}=1$ into (4.2) yields

$$
\begin{aligned}
\inf _{q_{1}} \quad\{- & h^{\prime}(T-t)-c \nu e^{r(T-t)}-\lambda_{l}-\lambda_{2}-\lambda+\left(\delta\left(q_{1}\right)+q_{1} a_{1}+a_{2}\right) \nu e^{r(T-t)} \\
+ & \left.\frac{1}{2}\left(\sigma_{1}^{2} q_{1}^{2}+\sigma_{2}^{2}+2 q_{1} \lambda \mu_{1} \mu_{2}\right) \nu^{2} e^{2 r(T-t)}\right\}=0
\end{aligned}
$$

for $t<T$. It can be shown that the minimizer of the equation (4.9) has the form

$$
\tilde{q}_{1}(T-t)=\frac{\eta_{1} a_{1} e^{-r(T-t)}-\lambda \mu_{1} \mu_{2} \nu}{\sigma_{1}^{2} \nu} .
$$

Let

$$
\tilde{t}_{03}=T-\frac{1}{r} \ln \left(\frac{\eta_{1} a_{1}}{\sigma_{1}^{2}+\lambda \mu_{1} \mu_{2}}\right) .
$$

For $t_{4} \leq t \leq \tilde{t}_{03}$, it is easy to see that $\left(q_{1 t}^{*}, q_{1 t}^{*}\right)=\left(\tilde{q}_{1}(T-t), 1\right)$. Putting $\left(\tilde{q}_{1}(T-t), 1\right)$ into $(4.2)$ gives

$$
h_{7}(T-t)=\tilde{h}_{7}(T-t)+C_{7},
$$

where

$$
\begin{aligned}
\tilde{h}_{7}(T-t)= & \frac{1}{r}\left(\left(1+\eta_{1}\right) a_{1}+a_{2}-c\right) \nu e^{r(T-t)}+\frac{1}{4 r} \sigma_{2}^{2} \nu^{2} e^{2 r(T-t)} \\
& -\int_{0}^{T-t} \eta_{1} \tilde{q}_{1}(s) a_{1} \nu e^{r s}-\frac{1}{2}\left(\sigma_{1}^{2} \tilde{q}_{1}^{2}(s)+2 \tilde{q}_{1}(s) \lambda \mu_{1} \mu_{2}\right) \nu^{2} e^{2 r s} d s
\end{aligned}
$$

For $\tilde{t}_{03} \leq t \leq T$, it follows that $\left(q_{1 t}^{*}, q_{1 t}^{*}\right)=(1,1)$. Plugging this into $(4.2)$, we get

$$
\begin{aligned}
h_{8}(T-t)= & \frac{1}{r}\left(a_{1}+a_{2}-c\right) \nu\left(e^{r(T-t)}-1\right) \\
& +\frac{1}{4 r}\left(\sigma_{1}^{2}+\sigma_{2}^{2}+2 \lambda \mu_{1} \mu_{2}\right) \nu^{2}\left(e^{2 r(T-t)}-1\right) .
\end{aligned}
$$

Let

$$
C_{6}=h_{7}\left(T-t_{4}\right)-\tilde{h}_{6}\left(T-t_{4}\right), \quad \text { and } \quad C_{7}=h_{8}\left(T-\tilde{t}_{03}\right)-\tilde{h}_{7}\left(T-\tilde{t}_{03}\right) .
$$

Then, we have

$$
\left\{\begin{array}{l}
h_{7}\left(T-t_{4}\right)=\tilde{h}_{6}\left(T-t_{4}\right)+C_{6}=h_{6}\left(T-t_{4}\right), \\
h_{8}\left(T-\tilde{t}_{03}\right)=\tilde{h}_{7}\left(T-\tilde{t}_{03}\right)+C_{7}=h_{7}\left(T-\tilde{t}_{03}\right) .
\end{array}\right.
$$

Case II: In this case, we still have $\bar{q}_{1}(T-t)>0$ and $\bar{q}_{2}(T-t)>0$ but $A_{1} \geq A_{2}$. Thus, $t_{3} \leq t_{4}$. For $0 \leq t \leq t_{3},\left(q_{1 t}^{*}, q_{2 t}^{*}\right)=\left(\bar{q}_{1}(T-t), \bar{q}_{2}(T-t)\right)$. This together with (4.2) gives

$$
h_{9}(T-t)=\tilde{h}_{6}(T-t)+C_{9}
$$


For $t \geq t_{3}$, we have $\bar{q}_{1}(T-t) \geq 1$, and thus $q_{1 t}^{*}=1$. By substituting $q_{1 t}^{*}=1$ into $(4.2)$ and mimicking the derivation of $(4.10)$, we obtain the minimizer

$$
\tilde{q}_{2}(T-t)=\frac{\eta_{2} a_{2} e^{-r(T-t)}-\lambda \mu_{1} \mu_{2} \nu}{\sigma_{2}^{2} \nu} .
$$

Write

$$
\tilde{t}_{04}=T-\frac{1}{r} \ln \left(\frac{\eta_{2} a_{2}}{\left(\sigma_{2}^{2}+\lambda \mu_{1} \mu_{2}\right) \nu}\right) .
$$

It is easy to check that $\tilde{t}_{04} \geq t_{3}$. For $t_{3} \leq t \leq \tilde{t}_{04},\left(q_{1 t}^{*}, q_{1 t}^{*}\right)=\left(1, \tilde{q}_{2}(T-t)\right)$. Putting $\left(1, \tilde{q}_{2}(T-t)\right)$ into (4.2), we obtain

$$
h_{10}(T-t)=\tilde{h}_{10}(T-t)+C_{10}
$$

where

$$
\begin{aligned}
\tilde{h}_{10}(T-t)= & \frac{1}{r}\left(\left(1+\eta_{2}\right) a_{2}+a_{1}-c\right) \nu e^{r(T-t)}+\frac{1}{4 r} \sigma_{1}^{2} \nu^{2} e^{2 r(T-t)} \\
& -\int_{0}^{T-t} \eta_{2} \tilde{q}_{2}(s) a_{2} \nu e^{r s}-\frac{1}{2}\left(\sigma_{2}^{2} \tilde{q}_{2}^{2}(s)+2 \tilde{q}_{2}(s) \lambda \mu_{1} \mu_{2}\right) \nu^{2} e^{2 r s} d s .
\end{aligned}
$$

For $\tilde{t}_{04} \leq t \leq T,\left(q_{1 t}^{*}, q_{1 t}^{*}\right)=(1,1)$. Then, it can be shown that $h(T-t)$ function in $(4.2)$ is given by $h_{8}$ of (4.12).

Let

$$
C_{9}=h_{10}\left(T-t_{3}\right)-\tilde{h}_{6}\left(T-t_{3}\right), \quad \text { and } \quad C_{10}=h_{8}\left(T-\tilde{t}_{04}\right)-\tilde{h}_{10}\left(T-\tilde{t}_{04}\right) .
$$

Then, we have

$$
\left\{\begin{array}{l}
h_{10}\left(T-t_{3}\right)=\tilde{h}_{6}\left(T-t_{3}\right)+C_{9}=h_{9}\left(T-t_{3}\right), \\
h_{8}\left(T-\tilde{t}_{04}\right)=\tilde{h}_{10}\left(T-\tilde{t}_{04}\right)+C_{10}=h_{10}\left(T-\tilde{t}_{04}\right) .
\end{array}\right.
$$

Case III: In this case, we have $\bar{q}_{1}(T-t)<0$ and $\bar{q}_{2}(T-t)>0$, and thus $q_{1 t}^{*}=0$. Substituting $q_{1 t}^{*}=0$ into $(4.2)$ yields the minimizer

$$
\check{q}_{2}(T-t)=\frac{\eta_{2} a_{2}}{\sigma_{2}^{2} \nu} \cdot e^{-r(T-t)}
$$

Let

$$
t_{04}=T-\frac{1}{r} \ln \left(\frac{\eta_{2} a_{2}}{\sigma_{2}^{2} \nu}\right)
$$

For $0 \leq t \leq t_{04}$, it follows that $\left(q_{1 t}^{*}, q_{1 t}^{*}\right)=\left(0, \breve{q}_{2}(T-t)\right)$. Plugging $\left(0, \check{q}_{2}(T-t)\right)$ into $(4.2)$, we get

$$
h_{11}(T-t)=\tilde{h}_{11}(T-t)+C_{11}
$$


where

$$
\tilde{h}_{11}(T-t)=\frac{1}{r}\left(\left(1+\eta_{2}\right) a_{2}+\left(1+\eta_{1}\right) a_{1}-c\right) \nu e^{r(T-t)}-\frac{1}{4 r} \frac{\eta_{2}^{2} a_{2}^{2}}{\sigma_{2}^{2}}(T-t) .
$$

For $t_{04} \leq t \leq T,\left(q_{1 t}^{*}, q_{2 t}^{*}\right)=(0,1)$. Using these optimal values and (4.2), we obtain

$$
h_{12}(T-t)=\frac{1}{r}\left(a_{2}+\left(1+\eta_{1}\right) a_{1}-c\right) \nu\left(e^{r(T-t)}-1\right)+\frac{1}{4 r} \sigma_{2}^{2} \nu^{2}\left(e^{2 r(T-t)}-1\right) .
$$

Let $C_{11}=h_{12}\left(T-t_{04}\right)-\tilde{h}_{11}\left(T-t_{04}\right)$. Then, we have

$$
h_{11}\left(T-t_{04}\right)=\tilde{h}_{11}\left(T-t_{04}\right)+C_{11}=h_{12}\left(T-t_{04}\right) .
$$

Case IV: In this case, we have $\bar{q}_{1}(T-t)>0$ and $\bar{q}_{2}(T-t)<0$, and thus $q_{2 t}^{*}=0$. Substituting $q_{2 t}^{*}=0$ into (4.2) yields the minimizer

$$
\check{q}_{1}(T-t)=\frac{\eta_{1} a_{1}}{\sigma_{1}^{2} \nu} \cdot e^{-r(T-t)} .
$$

Write

$$
t_{03}=T-\frac{1}{r} \ln \left(\frac{\eta_{1} a_{1}}{\sigma_{1}^{2} \nu}\right)
$$

For $0 \leq t \leq t_{03},\left(q_{1 t}^{*}, q_{1 t}^{*}\right)=\left(\check{q}_{1}(T-t), 0\right)$. This together with (4.2) yields

$$
h_{13}(T-t)=\tilde{h}_{13}(T-t)+C_{13},
$$

where

$$
\tilde{h}_{13}(T-t)=\frac{1}{r}\left(\left(1+\eta_{2}\right) a_{2}+\left(1+\eta_{1}\right) a_{1}-c\right) \nu e^{r(T-t)}-\frac{1}{4 r} \frac{\eta_{1}^{2} a_{1}^{2}}{\sigma_{1}^{2}}(T-t) .
$$

For $t_{03} \leq t \leq T$, it follows that $\left(q_{1 t}^{*}, q_{2 t}^{*}\right)=(1,0)$. Again, putting this into $(4.2)$, we get

$$
h_{14}(T-t)=\frac{1}{r}\left(a_{1}+\left(1+\eta_{2}\right) a_{2}-c\right) \nu\left(e^{r(T-t)}-1\right)+\frac{1}{4 r} \sigma_{1}^{2} \nu^{2}\left(e^{2 r(T-t)}-1\right) .
$$

Let $C_{13}=h_{14}\left(T-t_{04}\right)-\tilde{h}_{13}\left(T-t_{03}\right)$. Then, we have

$$
h_{13}\left(T-t_{03}\right)=\tilde{h}_{13}\left(T-t_{03}\right)+C_{13}=h_{14}\left(T-t_{03}\right) .
$$

To summarize, we state the results in the following theorem.

Theorem 4.1. Let $\left(\bar{q}_{1}(T-t), \bar{q}_{2}(T-t)\right)$, $\tilde{q}_{1}(T-t), \tilde{q}_{2}(T-t), \check{q}_{2}(T-t)$, and $\check{q}_{1}(T-t)$ be given in (4.5), (4.10), (4.14), (4.16) and (4.19), respectively. Also, recall the functions $h_{i}(T-t)$ for $i=6,7, \ldots, 14$ given in (4.8), (4.11), (4.12), (4.13), (4.15), (4.17), (4.18), (4.20) and (4.21), respectively. Then, we have 
(i) If Case I holds, then the optimal reinsurance strategies under the model (2.2) are

$$
\left(\bar{q}_{1 t}^{*}, \bar{q}_{2 t}^{*}\right)= \begin{cases}\left(\bar{q}_{1}(T-t), \bar{q}_{2}(T-t)\right), & 0 \leq t \leq t_{4}, \\ \left(\tilde{q}_{1}(T-t), 1\right), & t_{4} \leq t \leq \tilde{t}_{03}, \\ (1,1), & \tilde{t}_{03} \leq t \leq T\end{cases}
$$

for any $t \in[0, T]$, and the value function is given by

$$
V(t, x)= \begin{cases}-\frac{\varrho}{\nu} \exp \left\{-\nu x e^{r(T-t)}+h_{6}(T-t)\right\}, & 0 \leq t \leq t_{4}, \\ -\frac{\varrho}{\nu} \exp \left\{-\nu x e^{r(T-t)}+h_{7}(T-t)\right\}, & t_{4} \leq t \leq \tilde{t}_{03}, \\ -\frac{\varrho}{\nu} \exp \left\{-\nu x e^{r(T-t)}+h_{8}(T-t)\right\}, & \tilde{t}_{03} \leq t \leq T\end{cases}
$$

(ii) If Case II holds, then the optimal reinsurance strategies under the model (2.2) are

$$
\left(\bar{q}_{1 t}^{*}, \bar{q}_{2 t}^{*}\right)= \begin{cases}\left(\bar{q}_{1}(T-t), \bar{q}_{2}(T-t)\right), & 0 \leq t \leq t_{3} \\ \left(1, \tilde{q}_{2}(T-t)\right), & t_{3} \leq t \leq \tilde{t}_{04}, \\ (1,1), & \tilde{t}_{04} \leq t \leq T\end{cases}
$$

for any $t \in[0, T]$, and the value function is given by

$$
V(t, x)= \begin{cases}-\frac{\varrho}{\nu} \exp \left\{-\nu x e^{r(T-t)}+h_{9}(T-t)\right\}, & 0 \leq t \leq t_{3}, \\ -\frac{\varrho}{\nu} \exp \left\{-\nu x e^{r(T-t)}+h_{10}(T-t)\right\}, & t_{3} \leq t \leq \tilde{t}_{04}, \\ -\frac{\varrho}{\nu} \exp \left\{-\nu x e^{r(T-t)}+h_{8}(T-t)\right\}, & \tilde{t}_{04} \leq t \leq T ;\end{cases}
$$

(iii) If Case III holds, then the optimal reinsurance strategies under the model (2.2) are

$$
\left(\bar{q}_{1 t}^{*}, \bar{q}_{2 t}^{*}\right)= \begin{cases}\left(0, \check{q}_{2}(T-t)\right), & 0 \leq t \leq t_{04}, \\ (0,1), & t_{04} \leq T\end{cases}
$$

for any $t \in[0, T]$, and the value function is given by

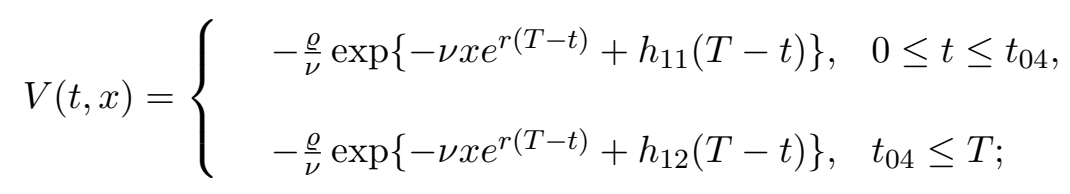


(iv) If Case VI holds, then the optimal reinsurance strategies under the model (2.2) are

$$
\left(\bar{q}_{1 t}^{*}, \bar{q}_{2 t}^{*}\right)= \begin{cases}\left(\check{q}_{1}(T-t), 0\right), & 0 \leq t \leq t_{03}, \\ (1,0), & t_{03} \leq T,\end{cases}
$$

for any $t \in[0, T]$, and the value function is given by

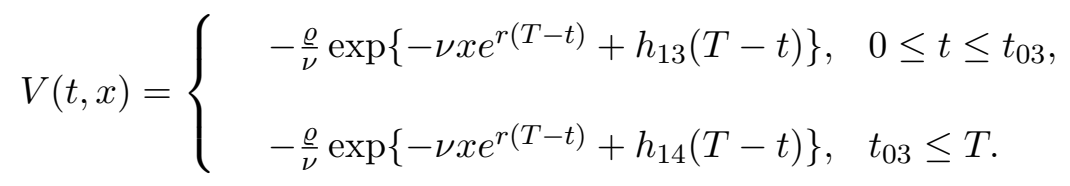

Remark 4.1. Since

$$
\begin{cases}h_{6}\left(T-t_{4}\right)=h_{7}\left(T-t_{4}\right), & h_{7}\left(T-\tilde{t}_{03}\right)=h_{8}\left(T-\tilde{t}_{03}\right), \\ h_{9}\left(T-t_{3}\right)=h_{10}\left(T-t_{3}\right), & h_{10}\left(T-\tilde{t}_{04}\right)=h_{8}\left(T-\tilde{t}_{04}\right), \\ h_{11}\left(T-t_{04}\right)=h_{12}\left(T-t_{04}\right), & h_{13}\left(T-t_{03}\right)=h_{14}\left(T-t_{03}\right),\end{cases}
$$

$V(t, x)$ is a continuous function for any $(t, x) \in[0, T] \times R$. Furthermore,

$$
\begin{cases}h_{6}^{\prime}\left(T-t_{4}\right)=h_{7}^{\prime}\left(T-t_{4}\right), & h_{7}^{\prime}\left(T-\tilde{t}_{03}\right)=h_{8}^{\prime}\left(T-\tilde{t}_{03}\right), \\ h_{9}^{\prime}\left(T-t_{3}\right)=h_{10}^{\prime}\left(T-t_{3}\right), & h_{10}^{\prime}\left(T-\tilde{t}_{04}\right)=h_{8}^{\prime}\left(T-\tilde{t}_{04}\right), \\ h_{11}^{\prime}\left(T-t_{04}\right)=h_{12}^{\prime}\left(T-t_{04}\right), & h_{13}^{\prime}\left(T-t_{03}\right)=h_{14}^{\prime}\left(T-t_{03}\right) .\end{cases}
$$

That is, $V(t, x)$ is a classical solution to the HJB equation (4.1).

Remark 4.2. Similar to the optimal results in the compound Poisson risk model, we can see from (4.5) that the optimal reinsurance strategies under the expected value principle in the diffusion risk model depend on the safety loading, time, interest rate as well as the claim size distributions and the counting processes. However, when we consider the model (2.2) under the variance principle, the results are very different. The optimal reinsurance strategies under the variance principle are always the same, and depend only on the safety loading, time and interest rate only (see (6.3) or Section 4 of Liang and Yuen (2014) for details).

Remark 4.3. In Sections 3 and 4, we show how the optimal strategies and value functions can be obtained for $m=2$. It is believed that the ideas and techniques shown here are still useful for deriving optimal results for $m>2$. However, when $m$ gets bigger, the number of cases needed to be considered may increase geometrically, and hence the optimal problem becomes very challenging. 


\section{$5 \quad$ Numerical examples}

In this section, we restrict our attention to the model with two dependent classes of insurance business under the expected value principle. For numerical examples using the variance principle, we refer the readers to Liang and Yuen (2014).

In Examples 5.1-5.3, we assume that the claim sizes $\left\{X_{i}\right\}$ and $\left\{Y_{i}\right\}$ are exponentially distributed with parameters $\alpha_{1}$ and $\alpha_{2}$, respectively. That is, $X \sim \exp \left(\alpha_{1}\right)$ and $Y \sim \exp \left(\alpha_{2}\right)$. For computational convenience, we set $\eta_{1}=\eta_{2}=\eta$, and hence

$$
\left\{\begin{aligned}
M_{1}\left(\nu q_{1} e^{r(T-t)}\right) & =\frac{\alpha_{1}}{\alpha_{1}-\nu q_{1} e^{r(T-t)}} \\
M_{2}\left(\nu q_{2} e^{r(T-t)}\right) & =\frac{\alpha_{2}}{\alpha_{2}-\nu q_{2} e^{r(T-t)}}
\end{aligned}\right.
$$

The minimizer $\left(q_{1}(T-t), q_{2}(T-t)\right)$ of $(3.3)$ in the compound Poisson model satisfies the following equations

$$
\left\{\begin{array}{l}
-(1+\eta)\left(\lambda_{1}+\lambda\right) / \alpha_{1}+\frac{\alpha_{1}}{\left(\alpha_{1}-\nu q_{1} e^{r(T-t)}\right)^{2}}\left(\lambda_{1}+\frac{\lambda \alpha_{2}}{\alpha_{2}-\nu q_{2} e^{r(T-t)}}\right)=0 \\
-(1+\eta)\left(\lambda_{2}+\lambda\right) / \alpha_{2}+\frac{\alpha_{2}}{\left(\alpha_{2}-\nu q_{2} e^{r(T-t)}\right)^{2}}\left(\lambda_{2}+\frac{\lambda \alpha_{1}}{\alpha_{1}-\nu q_{1} e^{r(T-t)}}\right)=0
\end{array}\right.
$$

and the minimizer $\left(\bar{q}_{1}(T-t), \bar{q}_{2}(T-t)\right)$ of $(4.2)$ in the diffusion case has the form as

$$
\left\{\begin{array}{l}
\bar{q}_{1}(T-t)=\frac{\alpha_{1}\left(2\left(\lambda_{1}+\lambda\right)\left(\lambda_{2}+\lambda\right)-\lambda\left(\lambda_{2}+\lambda\right)\right)}{4\left(\lambda_{1}+\lambda\right)\left(\lambda_{2}+\lambda\right)-\lambda^{2}} \cdot \frac{\eta}{\nu} e^{-r(T-t)}, \\
\bar{q}_{2}(T-t)=\frac{\alpha_{2}\left(2\left(\lambda_{1}+\lambda\right)\left(\lambda_{2}+\lambda\right)-\lambda\left(\lambda_{1}+\lambda\right)\right)}{4\left(\lambda_{1}+\lambda\right)\left(\lambda_{2}+\lambda\right)-\lambda^{2}} \cdot \frac{\eta}{\nu} e^{-r(T-t)} .
\end{array}\right.
$$

Besides, for the diffusion risk model, we have

$$
\left\{\begin{array}{lll}
\bar{q}_{2}^{*}=\frac{\eta\left(\lambda_{1}+\lambda\right) e^{-r(T-t)}-\frac{\lambda}{\alpha_{2}} \nu}{\left(\lambda_{1}+\lambda\right) \frac{2}{\alpha_{1}} \nu}, & \text { when } \quad \bar{q}_{1}^{*}=1 ; \\
\bar{q}_{1}^{*}=\frac{\eta\left(\lambda_{2}+\lambda\right) e^{-r(T-t)}-\frac{\lambda}{\alpha_{1}} \nu}{\left(\lambda_{2}+\lambda\right) \frac{2}{\alpha_{2}} \nu}, & \text { when } \quad \bar{q}_{2}^{*}=1 .
\end{array}\right.
$$

Example 5.1. Let $\eta=2, r=0.3, T=10, \lambda_{1}=3, \lambda_{2}=4, \lambda=2, \nu=0.5$, and $t=2.5$. The results are shown in Table 5.1 . 
Table 5.1 The effect of $\alpha_{1}$ on the optimal reinsurance strategies

\begin{tabular}{ccccccccc}
\hline \hline$\alpha_{1}$ & $t_{1}$ & $t_{2}$ & $q_{1}^{*}$ & $q_{2}^{*}$ & $t_{3}$ & $t_{4}$ & $\bar{q}_{1}^{*}$ & $\bar{q}_{2}^{*}$ \\
\hline 1 & 10 & 7.3303 & 0.0755 & 0.2348 & 8.3203 & 4.5222 & 0.1745 & 0.5452 \\
2 & 8.8024 & 7.3303 & 0.1510 & 0.2348 & 6.0098 & 4.5222 & 0.3489 & 0.5452 \\
3 & 7.4508 & 7.3303 & 0.2264 & 0.2348 & 4.6582 & 4.5222 & 0.5234 & 0.5452 \\
4 & 6.4919 & 7.3303 & 0.3019 & 0.2348 & 3.6993 & 4.5222 & 0.6978 & 0.5452 \\
5 & 5.7481 & 7.3303 & 0.3774 & 0.2348 & 2.9555 & 4.5222 & 0.8723 & 0.5452 \\
6 & 5.1403 & 7.3303 & 0.4529 & 0.2348 & 2.3477 & 4.5222 & 1 & $\mathbf{0 . 5 4 9 1}$ \\
7 & 4.6265 & 7.3303 & 0.5284 & 0.2348 & 1.8339 & 4.5222 & 1 & $\mathbf{0 . 5 6 1 0}$ \\
\hline
\end{tabular}

From Tables $5.1\left(\alpha_{2}=3\right)$, we can see that a greater value of $\alpha_{1}$ yields greater values of the optimal reinsurance strategies $q_{1}^{*}\left(\bar{q}_{1}^{*}\right)$. As expected, since a smaller value of $\alpha_{1}$ implies "more risky" claim size with a larger expected value, the insurer would rather retain a less share of each claim. Also, it is easy to observe from the tables that $q_{2}^{*}\left(\bar{q}_{2}^{*}\right)$ is independent of $\alpha_{1}$. That is, when the claim sizes are exponentially distributed, $X(Y)$ has no effect on the optimal reinsurance strategies $q_{2}^{*}$ and $\bar{q}_{2}^{*}\left(q_{1}^{*}\right.$ and $\left.\bar{q}_{1}^{*}\right)$. This interesting phenomenon may only appear in the case with exponential claim sizes and the reinsurance premium calculated under the expected value principle. This guess is partly due to the following two facts: (i) it was shown in Tables 4 and 5 in Liang and Yuen (2014) that the optimal results under the variance principle are indeed affected by both claim size distributions in the compound Poisson risk model; (ii) we see later in Example 5.4 and Table 5.4 that the claim sizes $Y(X)$ does affect the values of the optimal reinsurance strategies $q_{2}^{*}$ and $\bar{q}_{2}^{*}$ as well as $q_{1}^{*}$ and $\bar{q}_{1}^{*}$, when $Y(X)$ follows a gamma distribution with parameters $\beta \neq 1$ and $\alpha$. However, when one of the optimal strategies hits the boundary one, we see from the bold-faced values in Table 5.1 that the other optimal strategy is forced to change regardless to the choice of the claim size distributions. Moreover, we can see from Tables 5.1 that the value of $q_{1}^{*}\left(\bar{q}_{1}^{*}\right)$ is always smaller than the value of $q_{2}^{*}\left(\bar{q}_{2}^{*}\right)$ when the inequality $\alpha_{1}<\alpha_{2}$ holds, and vice versa. This suggests that the values of the optimal reinsurance strategies are more sensitive to the claim size distribution than to the counting processes (see Table 5.3). 
Remark 5.1. The bold-faced values in Tables 5.1-5.3 are calculated using (5.1) when one of the optimal reinsurance strategies is 1 .

Example 5.2. Let $r=0.3, T=10, \lambda_{2}=4, \eta=2, \nu=0.5, \alpha_{1}=2, \alpha_{2}=3$, and $t=4.6$. The results are shown in Table 5.2.

From Table 5.2, we see that the optimal reinsurance strategies decrease while $\lambda$ increases. Since a greater value of $\lambda$ implies a greater value of expected claim number, the insurer would rather retain a less share of each claim.

Table 5.2 The effect of $\lambda$ on the optimal reinsurance strategies

\begin{tabular}{ccccccccc}
\hline \hline$\lambda$ & $t_{1}$ & $t_{2}$ & $q_{1}^{*}$ & $q_{2}^{*}$ & $t_{3}$ & $t_{4}$ & $\bar{q}_{1}^{*}$ & $\bar{q}_{2}^{*}$ \\
\hline 1 & 8.6176 & 7.1790 & 0.2996 & 0.4613 & 5.7821 & 4.3367 & $\mathbf{0 . 7 0 8 3}$ & 1 \\
2 & 8.8024 & 7.3303 & 0.2835 & 0.4408 & 6.0098 & 4.5222 & $\mathbf{0 . 6 5 8 3}$ & 1 \\
3 & 8.9134 & 7.3678 & 0.2742 & 0.4359 & 6.1542 & 4.6478 & 0.6273 & 0.9858 \\
4 & 8.9871 & 7.4993 & 0.2682 & 0.4190 & 6.2537 & 4.7395 & 0.6089 & 0.9590 \\
5 & 9.0391 & 7.5526 & 0.2640 & 0.4124 & 6.3251 & 4.8095 & 0.5960 & 0.9391 \\
6 & 9.0778 & 7.5946 & 0.2610 & 0.4072 & 6.3796 & 4.8651 & 0.5863 & 0.9236 \\
7 & 9.1075 & 7.6286 & 0.2587 & 0.4031 & 6.4212 & 4.9105 & 0.5791 & 0.9110 \\
\hline
\end{tabular}

Example 5.3. Let $r=0.3, T=10, \lambda_{2}=4, \eta=2, \lambda=2, \nu=0.5, \alpha_{1}=2, \alpha_{2}=3$, and $t=4.5$. The results are shown in Table 5.3. 
Table 5.3 The effect of $\lambda_{1}$ on the optimal reinsurance strategies

\begin{tabular}{ccccccccc}
\hline \hline$\lambda_{1}$ & $t_{1}$ & $t_{2}$ & $q_{1}^{*}$ & $q_{2}^{*}$ & $t_{3}$ & $t_{4}$ & $\bar{q}_{1}^{*}$ & $\bar{q}_{2}^{*}$ \\
\hline 1 & 9.2321 & 7.2525 & 0.2418 & 0.4379 & 6.5400 & 4.4448 & $\mathbf{0 . 5 4 6 0}$ & 1 \\
2 & 8.9572 & 7.2998 & 0.2626 & 0.4317 & 6.1961 & 4.4935 & $\mathbf{0 . 6 0 1 5}$ & 1 \\
3 & 8.8024 & 7.3303 & 0.2751 & 0.4278 & 6.0098 & 4.5222 & 0.6358 & 0.9934 \\
5 & 8.6340 & 7.3672 & 0.2893 & 0.4231 & 5.8127 & 4.5549 & 0.6745 & 0.9837 \\
6 & 8.5832 & 7.3792 & 0.2938 & 0.4216 & 5.7540 & 4.5650 & 0.6865 & 0.9807 \\
7 & 8.5442 & 7.3886 & 0.2972 & 0.4204 & 5.7092 & 4.5729 & 0.6958 & 0.9784 \\
8 & 8.5134 & 7.3963 & 0.3000 & 0.4194 & 5.6740 & 4.5791 & 0.7031 & 0.9765 \\
\hline
\end{tabular}

From Table 5.3, we see that a greater value of $\lambda_{1}$ yields greater values of the optimal reinsurance strategies $q_{1}^{*}$ and $\bar{q}_{1}^{*}$ but smaller values of the optimal reinsurance strategy $q_{2}^{*}$ and $\bar{q}_{2}^{*}$. Along the same lines, one can numerically show that a greater value of $\lambda_{2}$ yields greater values of $q_{2}^{*}$ and $\bar{q}_{2}^{*}$ but smaller values of $q_{1}^{*}$ and $\bar{q}_{1}^{*}$.

In the following example, we show that $q_{1}^{*}\left(q_{2}^{*}\right)$ does depend on the claim sizes $Y(X)$ for some distributions. We assume that the claim sizes $Y$ has a gamma distribution with parameters $\beta \neq 1$ and $\alpha$, that is, $Y \sim \Gamma(\beta, \alpha)$. Then, we have

$$
\begin{cases}\mu_{2} & =E(Y)=\frac{\beta}{\alpha}, \\ \sigma_{2}^{2} & =\left(\lambda_{2}+\lambda\right)\left(\beta^{2}+\beta\right) / \alpha^{2}, \\ M_{2}(r) & =\left(\frac{\alpha}{\alpha-r}\right)^{\beta}, \text { for } \quad r<\alpha .\end{cases}
$$

The minimizer $\left(q_{1}(T-t), q_{2}(T-t)\right)$ of (3.3) for the compound Poisson model satisfies the following equations

$$
\begin{cases}-(1+\eta)\left(\lambda_{1}+\lambda\right) / \alpha_{1}+\frac{\alpha_{1}}{\left(\alpha_{1}-\nu q_{1} e^{r(T-t)}\right)^{2}}\left(\lambda_{1}+\lambda\left(\frac{\alpha}{\alpha-\nu q_{2} e^{r(T-t)}}\right)^{\beta}\right) & =0 \\ -(1+\eta)\left(\lambda_{2}+\lambda\right) \beta / \alpha+\beta\left(\frac{\alpha}{\alpha-\nu q_{2} e^{r(T-t)}}\right)^{\beta-1} \frac{\alpha}{\left(\alpha-\nu q_{2} e^{r(T-t)}\right)^{2}}\left(\lambda_{2}+\frac{\lambda \alpha_{1}}{\alpha_{1}-\nu q_{1} e^{r(T-t)}}\right) & =0\end{cases}
$$


and the minimizer $\left(\bar{q}_{1}(T-t), \bar{q}_{2}(T-t)\right)$ of $(4.2)$ for the diffusion model is given by

$$
\left\{\begin{array}{l}
\bar{q}_{1}(T-t)=\frac{\alpha_{1}\left(\left(\lambda_{1}+\lambda\right)\left(\lambda_{2}+\lambda\right)(\beta+1)-\lambda \beta\left(\lambda_{2}+\lambda\right)\right)}{2\left(\lambda_{1}+\lambda\right)\left(\lambda_{2}+\lambda\right)(\beta+1)-\lambda^{2} \beta} \cdot \frac{\eta}{\nu} e^{-r(T-t)}, \\
\bar{q}_{2}(T-t)=\frac{\alpha\left(2\left(\lambda_{1}+\lambda\right)\left(\lambda_{2}+\lambda\right)-\lambda\left(\lambda_{1}+\lambda\right)\right)}{2\left(\lambda_{1}+\lambda\right)\left(\lambda_{2}+\lambda\right)(\beta+1)-\lambda^{2} \beta} \cdot \frac{\eta}{\nu} e^{-r(T-t)}
\end{array}\right.
$$

Example 5.4. Assume that $X \sim \exp \left(\alpha_{1}\right)$ and $Y \sim \Gamma(\beta, \alpha)$. Let $\lambda=2, \lambda_{1}=3, r=0.3, T=10$, $\lambda_{2}=4, \eta=2, \nu=0.5, \alpha_{1}=2, \alpha=3$, and $t=1.8$. The results are shown in Table 5.4.

Table 5.4 The effect of $\beta$ on the optimal reinsurance strategies

\begin{tabular}{ccccccccc}
\hline \hline$\beta$ & $t_{1}$ & $t_{2}$ & $q_{1}^{*}$ & $q_{2}^{*}$ & $t_{3}$ & $t_{4}$ & $\bar{q}_{1}^{*}$ & $\bar{q}_{2}^{*}$ \\
\hline 0.6 & 8.6290 & 6.7988 & 0.1289 & 0.2232 & 5.8364 & 3.8070 & 0.2979 & 0.5477 \\
0.7 & 8.6769 & 6.9395 & 0.1271 & 0.2140 & 5,8861 & 4.0007 & 0.2935 & 0.5167 \\
0.8 & 8.7216 & 7.0747 & 0.1254 & 0.2055 & 5.9312 & 4.1837 & 0.2896 & 0.4891 \\
0.9 & 8.7633 & 7.2049 & 0.1238 & 0.1976 & 5.9723 & 4.3573 & 0.2860 & 0.4643 \\
1 & 8.8024 & 7.3303 & 0.1224 & 0.1903 & 6.0098 & 4.5222 & 0.2828 & 0.4419 \\
2 & 9.0886 & 8.3819 & 0.1123 & 0.1388 & 6.2613 & 5.8352 & 0.2623 & 0.2980 \\
3 & 9.2627 & 9.1830 & 0.1066 & 0.1092 & 6.3970 & 6.7747 & 0.2518 & 0.2248 \\
\hline
\end{tabular}

From Table 5.4, we see that a greater value of $\beta$ yields smaller values of the optimal reinsurance strategies $q_{i}^{*}$ and $\bar{q}_{i}^{*}(i=1,2)$. Since a greater value of $\beta$ implies "more risky" claim sizes with a larger expected value, the insurer would rather retain a less share of each claim in general. It is also reasonable to expect that the optimal reinsurance strategy $q_{2}^{*}\left(\bar{q}_{2}^{*}\right)$ decreases faster than $q_{1}^{*}$ $\left(\bar{q}_{1}^{*}\right)$ as the claim size distribution $Y$ has a more direct impact on $q_{2}^{*}\left(\bar{q}_{2}^{*}\right)$.

In the numerical examples, we find that the optimal reinsurance strategies are more sensitive to the claim size distribution than to the counting processes. This finding is consistent with that under the variance principle (see Tables 4-7 in Liang and Yuen (2014)). Moreover, when the claim 
sizes are exponentially distributed, under the expected value principle, the optimal reinsurance strategy of one class of insurance risk is independent of the claim sizes of the other class. However, this is not true when the reinsurance premium is calculated using the variance principle, under which both claim size distributions do have impact on the optimal reinsurance strategies of the two classes (see Tables 4 and 5 in Liang and Yuen (2014)). Furthermore, in the diffusion risk model, the optimal reinsurance strategies under the expected value principle depend on the safety loading, time, interest rate, the claim size distributions and the counting processes, while those under variance principle depend only on the safety loading, time and interest rate (see (6.3) below).

\section{$6 \quad$ Further results}

For the diffusion risk model, we now extend the work of Liang and Yuen (2014) to the case with $m>2$ classes of insurance business under the variance principle. In this case, the reinsurance premium rate becomes

$$
\delta(q)=\sum_{l=1}^{m}\left(1-q_{l}\right) a_{l}+\Lambda\left(\sum_{l=1}^{m}\left(1-q_{l}\right)^{2} \sigma_{l}^{2}+\sum_{l=1}^{m} \sum_{k=1(k \neq l)}^{m}\left(1-q_{l}\right)\left(1-q_{k}\right) \lambda \mu_{l} \mu_{k}\right),
$$

here $\Lambda$ is the reinsurer's safety loading. Following the steps in Section 4, one can show that the optimal reinsurance strategies for the diffusion risk model satisfy the following equations

$$
-2 \Lambda\left(\left(1-q_{l}\right) \sigma_{l}^{2}+\sum_{k=1(k \neq l)}^{m}\left(1-q_{k}\right) \lambda \mu_{l} \mu_{k}\right)+\left(q_{l} \sigma_{l}^{2}+\lambda \sum_{i \neq l} q_{i} \mu_{l} \mu_{i}\right) \nu e^{r(T-t)}=0,
$$

for $l=1,2, \ldots, m$; or equivalently,

$$
\mathbf{A} q^{\prime}=\tilde{\mathbf{B}} \frac{2 \Lambda}{2 \Lambda+\nu e^{r(T-t)}},
$$

where

$$
\tilde{\mathbf{B}}=\left(\begin{array}{l}
\sigma_{1}^{2}+\sum_{k \neq 1} \lambda \mu_{1} \mu_{k} \\
\sigma_{2}^{2}+\sum_{k \neq 2} \lambda \mu_{2} \mu_{k} \\
\sigma_{3}^{2}+\sum_{k \neq 3} \lambda \mu_{3} \mu_{k} \\
\vdots \\
\sigma_{m}^{2}+\sum_{k \neq m} \lambda \mu_{m} \mu_{k}
\end{array}\right) .
$$

Write $\mathbf{I}=(1,1, \ldots, 1)_{1 \times m}^{\prime}$. Since $\tilde{\mathbf{B}}=\mathbf{A I}$ and the matrix $\mathbf{A}$ is invertible, the equation (6.2) has a unique solution

$$
\begin{aligned}
\bar{q}(T-t) & =\left(\mathbf{A}^{-1} \tilde{\mathbf{B}} \frac{2 \Lambda}{2 \Lambda+\nu e^{r(T-t)}}\right)^{\prime} \\
& =\mathbf{I}^{\prime} \frac{2 \Lambda}{2 \Lambda+\nu e^{r(T-t)}}
\end{aligned}
$$


which implies that, for any $t \in[0, T]$, the optimal reinsurance strategies under the variance principle in the diffusion model are

$$
q_{1 t}^{*}=q_{2 t}^{*}=\cdots=q_{m t}^{*}=\frac{2 \Lambda}{2 \Lambda+\nu e^{r(T-t)}},
$$

which depend only on the safety loading, time and interest rate. This result is in line with the one in Theorems 4.1 and 5.2 of Liang et al. (2011). In addition, for the case with different safety loadings, the reader is referred to (6.1) of Liang and Yuen (2014) for which the optimal strategies also depend on the claim size distributions and the counting processes. Similar results for the optimal strategies should be expected in the case with $m>2$.

For optimization problems under the variance principle, results similar to (6.3) (see Liang et al. (2011), Liang and Yuen (2014), and the reference therein) are often derived. That is, a more complicated premium principle, namely the variance premium principle, results in a simpler form of optimal value, at least compared to the results derived under the expected value principle. This interesting observation is really difficult to explain. A rough guess is that the variance or the dependence is considered both in the premium rate and surplus process, the impact of the claim size distributions and the counting processes is somehow cancelled out in the process of derivation.

\section{Conclusion}

The optimization reinsurance problem has been around for many years, and hence many interesting and useful results can be found in the literature. In this paper, we give a notable extension of the study to the risk model with common shock dependence.

From an insurer's point of view, we consider the optimal proportional reinsurance strategy in a risk model with $m \geq 2$ dependent classes of insurance business, which generalizes the results of Liang and Yuen (2014). Under the criterion of maximizing the expected exponential utility, closed-form expressions for the optimal strategies and value function are given not only for the compound Poisson risk model but also for the diffusion approximation risk model. Our results show that the optimal reinsurance strategies under the expected value principle are very different from those under the variance principle in the diffusion case. In particular, the former depends not only on the safety loading, time and interest rate, but also on the claim size distributions and the counting processes, and the optimal strategies of the two classes do not have the same form. 


\section{Acknowledgements}

The authors would like to thank the anonymous reviewer and the Editor for their constructive comments and suggestions. The research of Kam Chuen Yuen was supported by the Research Grants Council of the Hong Kong Special Administrative Region, China (Project No. HKU 7057/13P), and the CAE 2013 research grant from the Society of Actuaries - any opinions, finding, and conclusions or recommendations expressed in this material are those of the authors and do not necessarily reflect the views of the SOA. The research of Zhibin Liang and Ming Zhou was supported by the National Natural Science Foundation of China (Grant No.11471165) and Jiangsu Natural Science Foundation (Grant No. BK20141442).

\section{References}

[1] Bai, L. Cai, J. and Zhou, M., 2013. Optimal reinsurance policies for an insurer with a bivariate reserve risk process in a dynamic setting. Insurance: Mathematics and Economics 53, 664-670.

[2] Bernard, C. and Tian, W. (2009). Optimal reinsurance arrangements under tail risk measures. Journal of Risk and Insurance 76(3), 709-725.

[3] Browne, S., 1995. Optimal investment policies for a firm with random risk process: exponential utility and minimizing the probability of ruin. Mathematics of Operations Research 20, 937-958.

[4] Cai, J. and Tan, K. (2007). Optimal retention for a stop-loss reinsurance under the VaR and CTE risk measures. ASTIN Bulletin 37(1), 93-112.

[5] Centeno, M. (1986). Measuring the effects of reinsurance by the adjustment coefficient. Insurance: Mathematics and Economics 5, 169-182.

[6] Centeno, M. (2002). Excess of loss reinsurance and Gerber's inequality in the Sparre Anderson model. Insurance: Mathematics and Economics 31, 415-427.

[7] Centeno, M., 2005. Dependent risks and excess of loss reinsurance. Insurance: Mathematics and Economics 37, 229-238.

[8] Cheung, K. C., Sung, K. C. J., Yam, S. C. P. and Yung, S. P., 2014. Optimal reinsurance under general law-invariant risk measures. Scandinavian Actuarial Journal 1, 72-91.

[9] Chi, Y. and Meng, H., 2014. Optimal reinsurance arrangements in the presence of two reinsurers. Scandinavian Actuarial Journal 5, 424-438.

[10] Fleming, W., Soner, H., 2006. Controlled Markov Processes and Viscosity Solutions. 2nd Ed. SpringerVerlag, NY.

[11] Gerber, H., 1979. An introduction to mathematical risk theory. Huebner Foundation Monograph. No. 8. 
[12] Grandell, J., 1991. Aspects of risk theory. Springer-verlag, New York.

[13] Hald, M. and Schmidli, H. (2004). On the maximization of the adjustment coefficient under proportioal reinsurance. ASTIN Bulletin 34, 75-83.

[14] Irgens, C. and Paulsen, J. (2004). Optimal control of risk exposure, reinsurance and investments for insurance portfolios. Insurance: Mathematics and Economics 35, 21-51.

[15] Kaluszka, M. (2001). Optimal reinsurance under mean-variance premium principles. Insurance: Mathematics and Economics 28, 61-67.

[16] Kaluszka, M. (2004). Mean-variance optimal reinsurance arrangements. Scandinavian Actuarial Journal, $28-41$.

[17] Liang, Z. and Guo, J. (2008). Upper bound for ruin probabilities under optimal investment and proportional reinsurance. Applied Stochastic Models in Business and Industry 24, 109-128.

[18] Liang, Z., Yuen, Kam C. and Guo, J., 2011. Optimal proportional reinsurance and investment in a stock market with Ornstein-Uhlenbeck process. Insuarnce: Mathematics and Economics 49, 207-215.

[19] Liang, Z., Yuen, Kam C., 2014. Optimal dynamic reinsurance with dependent risks: variance premium principle. Scandinavian Actuarial Journal, DOI: 10.1080/03461238.2014.892899.

[20] Ortega, J. M. and Rheinboldt, W. C., 1970. Iterative solution of nonlinear equations in several variables. Academic Press, New York.

[21] Promislow, D. and Young, V. (2005). Minimizing the probability of ruin when claims follow Brownian motion with drift. North American Actuarial Journal 9(3), 109-128.

[22] Schmidli, H. (2001). Optimal proportional reinsurance policies in a dynamic setting. Scandinavian Actuarial Journal, 55-68.

[23] Schmidli, H. (2002). On minimizing the ruin probability by investment and reinsurance. Annals of Applied Probability 12, 890-907.

[24] Wang, G. and Yuen, Kam C., 2005. On a correlated aggregate claims model with thinning-dependence structure. Insurance: Mathematics and Economics 36, 456-468.

[25] Wang, S., 1998. Aggregation of correlated risk portfolios: Models and algorithms, Proceedings of the Casualty Actuarial Society, vol. LXXXV, pp. 848-939.

[26] Yang, H., Zhang, L., 2005. Optimal investment for insurer with jump-diffusion risk process. Insurance: Mathematics and Economics 37, 615-634.

[27] Yuen, Kam C., Guo, J., Wu, X., 2002. On a correclated aggregate claim model with Poisson and Erlang risk process. Insurance: Mathematics and Economics 31, 205-214.

[28] Yuen, Kam C., Guo, J., Wu, X., 2006. On the first time of ruin in the bivariate compound Poisson model. Insurance: Mathematics and Economics 38, 298-308. 